\title{
X-Ray Specular Reflection Studies of Silicon Coated by Organic Monolayers (Alkylsiloxanes)
}

\section{Citation}

Tidswell, I. M., B. M. Ocko, Peter S. Pershan, S. R. Wasserman, G. M. Whitesides, and J. D. Axe. 1990. X-ray specular reflection studies of silicon coated by organic monolayers (alkylsiloxanes). Physical Review B 41(2): 1111-1128.

\section{Published Version}

doi:10.1103/PhysRevB.41.1111

\section{Permanent link}

http://nrs.harvard.edu/urn-3:HUL.InstRepos:10354234

\section{Terms of Use}

This article was downloaded from Harvard University's DASH repository, and is made available under the terms and conditions applicable to Other Posted Material, as set forth at http:// nrs.harvard.edu/urn-3:HUL.InstRepos:dash.current.terms-of-use\#LAA

\section{Share Your Story}

The Harvard community has made this article openly available.

Please share how this access benefits you. Submit a story.

\section{Accessibility}




\title{
X-ray specular reflection studies of silicon coated by organic monolayers (alkylsiloxanes)
}

\author{
I. M. Tidswell, B. M. Ocko, ${ }^{*}$ and P. S. Pershan \\ Division of Applied Sciences and Department of Physics, Harvard University, Cambridge, Massachusetts 02138
}

S. R. Wasserman and G. M. Whitesides

Department of Chemistry, Harvard University, Cambridge, Massachusetts 02138

J. D. Axe

Department of Physics, Brookhaven National Laboratory, Upton, New York 11973

(Received 3 October 1988; revised manuscript received 7 August 1989)

\begin{abstract}
$X$-ray specular reflectivity has been used to characterize the structure of silicon-silicon-oxide surfaces coated with chemisorbed hydrocarbon monolayer films (alkylsiloxanes). Using synchrotron radiation the reflectivity was followed over 9 orders of magnitude, from grazing incidence to an incident angle of $\phi \approx 6.5^{\circ}$, or $q=(4 \pi / \lambda) \sin (\phi)=0.8 \AA^{-1}$, allowing a spatial resolution of features approximately $\pi / 0.8 \approx 4.0 \AA$ along the surface normal. Analysis was performed by fitting the data to reflectivities calculated from models of the surface electron density and by calculating Patterson functions directly from the data. Although the measured reflectivities could be equally well described by different sets of model parameters, the electron densities calculated from these different parameters were remarkably alike. Inspection of the electron densities allowed identification of a layer of $\mathrm{SiO}_{2}(\approx 17-\AA$ thick$)$, a layer of head-group region where the alkylsiloxane adsorbs to the $\mathrm{SiO}_{2}$, and the hydrocarbon layer. Fitting the data also required that the various interfaces have different widths. The fact that the same local hydrocarbon density of $0.85 \mathrm{~g} / \mathrm{cm}^{3}$ was observed for both fully formed and partially formed monolayers with alkane chains of varying length excluded a model in which the partially formed monolayer was made up of separated islands of well-formed monolayers. Measurements before and after chemical reaction of a monolayer in which the alkyl chain was terminated by an olefinic group demonstrated the ability to use $x$-ray reflectivity to characterize chemical changes. The effects of radiation damage on these types of measurements are described.
\end{abstract}

\section{INTRODUCTION}

Although Compton demonstrated the phenomena of small-angle $\mathrm{X}$-ray specular reflectivity by $1922,{ }^{1}$ we are not aware of any serious attempts to use the technique to characterize material surfaces before Parratt's measurements on copper surfaces in $1954 .^{2}$ Unfortunately his work was seriously limited by both the low brilliance [i.e., photons $\left./\left(\sec \mathrm{mm}^{2} \operatorname{mrad}^{2} 0.1 \% \Delta \lambda / \lambda\right)\right]$ of the x-ray beams that were available at that time as well as by the difficulty in obtaining a sufficiently smooth surface. ${ }^{2}$ Improved surface preparation techniques and modern experimental methods have permitted study of a broad range of surfaces using conventional or rotating anode $x$-ray sources. Examples include studies of mercury and liquid-metal surfaces, ${ }^{3-6}$ of both coated and uncoated solid substrates, ${ }^{7-9}$ and of surfactant monolayers on the surface of water. ${ }^{10}$ The use of high-brilliance synchrotron radiation by Als-Nielsen, Christensen, and Pershan to study specular reflectivity from the surface of the nematic liquid-crystal 4-cyano-4'-n-octyloxybiphenol (8OCB) greatly enhanced the utility of $x$-ray specular reflection as a probe of interface and surface structure by increasing the range of accessible scattering angles. ${ }^{11}$
Since then, a number of studies on surface of liquid crystals, ${ }^{12-16}$ microemulsions, ${ }^{17}$ simple liquids, ${ }^{18,19}$ insoluble monolayers on water, ${ }^{20-22}$ and metallic single crystals ${ }^{23,24}$ have followed. In most cases the methods by which the reflectivity was analyzed to obtain structural information were relatively simple. While these procedures are adequate for many surfaces, they were not adequate for the more complex surfaces to be discussed here.

In this paper we will describe measurements of $x$-ray reflectivity from silicon wafers coated with various alkylsiloxanes (i.e., alkylsilanes, $R\left(\mathrm{CH}_{2}\right)_{n} \mathrm{SiO}_{3}$, covalently bonded to the silicon wafer surface by oxygen-silicon bonds at the head of the chain with $R$ being one of severval moeities) using the technique of self-assembly. Specular reflectivity from the air-hydrocarbon, hydrocarbon-silicon-oxide, and the silicon-oxidecrystalline-silicon interfaces interferes to produce a combined reflectivity that is strongly dependent on the angle of incidence and the surface structure. By comparing calculated reflectivities from different models and by comparing the models with Patterson functions calculated directly from the data, we believe that we have been able to establish both the uniqueness and the confidence 
limits for a number of features of monolayers at the surface of silicon. These include the thicknesses of the hydrocarbon layer and the layer of silicon oxide between the hydrocarbon and the single crystal substrate, the widths of the interfaces between the various layers and the electron densities within each layer.

\section{Alkylsiloxane coated surfaces}

Most synthesis of organic monolayer films follows one of two different approaches. The first high-quality monolayer films, produced by Blodgett and Langmuir, were made by dipping a substrate into a trough of water coated with a monolayer organic film on the surface. ${ }^{25}$ Each pass of the substrate through the surface of the water applies a coat of either one or two monolayers, depending on the specific structure of the monolayer. A second technique, forming generally more rugged monolayers, makes use of certain molecules which, in solution, spontaneously assemble to form uniform monolayer coatings on solid surfaces. A full review of the production, characterization, and technological value of these and other types of organic thin films, together with extensive references to the literature, is given in the review by Swalen et al. ${ }^{26}$

The present studies are concerned with monolayers that form spontaneously on the surface of silicon-silicon-oxide substrates on immersion of the sample in dilute anhydrous solutions of alkyltrichlorosilanes of the form $\mathrm{Cl}_{3} \mathrm{Si}-\left(\mathrm{CH}_{2}\right)_{n}-R$, with $n$ varying from 9 to 17. In the simplest case, $R$ is the methyl group $\left(-\mathrm{CH}_{3}\right)$, but we have also studied molecules in which the terminal groups were $-\mathrm{CH}=\mathrm{CH}_{2}$ and $-\mathrm{CHBr}-\mathrm{CH}_{2} \mathrm{Br}$. We also measured the reflectivity of a monolayer prepared from a fluorocarbon of the structure $\mathrm{Cl}_{3} \mathrm{Si}-\left(\mathrm{CH}_{2}\right)_{2}-\left(\mathrm{CF}_{2}\right)_{7}-\mathrm{CF}_{3}$. Little experimental data exist on the formation of the alkylsiloxane film. ${ }^{27}$ On immersion of the silicon wafer into the trichlorosilane solution, the silicon-chlorine bonds of the head group on the molecule react with the surface hydroxyl groups of the silicon-oxide surface and with adsorbed water. This reaction results in a network of covalent $\mathrm{Si}-\mathrm{O}-\mathrm{Si}$ linkages that anchor the alkylsilyl moeity to the surface and to other alkylsilyls. The hydrocarbon film is therefore chemisorbed to the surface, in contrast to LangmuirBlodgett films which are generally bound to the substrate through much weaker hydrogen bonds and van der Waals interactions (physisorption). As a result, alkylsiloxane monolayers are much more rugged and resistant to chemical attack than are Langmuir-Blodgett films.

Studies on alkylsiloxane monolayers, of the form $-\mathrm{Si}-\left(\mathrm{CH}_{2}\right)_{n}-\mathrm{CH}_{3}$ with $n=12-20$, that have been reported by Sagiv in a series of papers over the last decade $^{8,28-32}$ confirm their high stability and general resistance to chemical attack. The high contact angles that have been measured for all of these surfaces with water $\left(110^{\circ}-115^{\circ}\right)$ and hexadecane $\left(38^{\circ}-45^{\circ}\right)$ indicate that they have low surface energies and are not prone to contamination through physisorption of airborne hydrocarbons or water. ${ }^{33}$ For comparison, clean silicon oxide surfaces which are wet by water have relatively high surface energy and readily adsorb airborne contaminants.
Ellipsometric measurements of well-formed monolayers are consistent with relatively dense packing of the alkyl groups and a mean thickness that equals (within experimental uncertainty) ${ }^{34}$ the theoretical length of the fully extended alkane chain. This thickness could, however, also be consistent with a molecular tilt away from the surface normal by as much as $15^{\circ}$. Contact angle studies also support the interpretation of relatively dense wellformed monolayers. ${ }^{30}$ Both the chemical stability and the high surface uniformity make alkylsiloxane monolayers ideal for study.

The limited brilliance from the rotating anode $x$-ray source used in the previous study of the $x$-ray reflectivity from alkylsiloxane monolayers ${ }^{8}$ restricted the range over which measurements could be taken to incident angles below $3^{\circ}$ (corresponding to about $0.4 \AA^{-1}$ ). As a result, only the overall thickness of the adsorbed monolayer could be obtained with any confidence. The length measured was about $13 \%$ less than the length of a fully extended layer. This was explained in terms of an average area per molecule of $20 \AA^{2}$ and an associated tilt of each molecule of about $30^{\circ}$ [e.g., $\cos ^{-1}(0.87) \approx 30^{\circ}$ ]. An unusually small width of $0.25 \AA$ was inferred for the alkyl-air and the silicon-oxide-alkyl interfaces, both being assigned the same width. Since these data were taken for a small range of incident angles, determination of narrow interface width is very difficult, and we believe this estimate to be significantly too small. In the study reported here, the use of synchrotron radiation made it possible to measure the reflectivity out to incident angles of the order of $7^{\circ}$, allowing a more accurate determination of the interface widths.

\section{REFLECTIVITY THEORY}

Even though the wavelength $\lambda$ is comparable to atomic dimensions, and consequently comparable to the roughness of the surface, specular reflection of $x$ rays can be described by the Fresnel laws of classical optics. ${ }^{19,35}$ The insert to Fig. 1 shows the kinematics for specular reflection of monochromatic $x$ rays from the surface of a solid. The refractive index of matter for $x$ rays of wavelength $\lambda$ is given by $n=1-\delta+i \beta$ where $\delta \approx \rho \lambda^{2} r_{0} / 2 \pi, \rho$ is the effective electron density, $r_{0}$ the classical electron radius or the Thompson scattering length, and $\beta \approx \lambda / 4 \pi \mu$ where $\mu$ is the $x$-ray absorption length. For the $x$-ray wavelengths of interest, both $\delta$ and $\beta$ are much less than 1. The effective electron density $\rho$ for low $-Z$ materials is just the total electron density of the material $\rho_{T}$. For materials where some fraction $f$ of the electrons has binding energies that are greater than the incident $x$-ray energy $\rho \approx \rho_{T}(1-f) . \quad$ Defining a critical angle $\phi_{c} \approx \sqrt{2} \delta$ $=\lambda \sqrt{\rho r_{0} / \pi}$ and using the classical Maxwell's equations yield the expression for the Fresnel reflectivity (from a sharp interface) at small angles $\phi,{ }^{36}$

$$
R_{F}(\phi) \approx\left|\frac{\phi-\left(\phi^{2}-\phi_{c}^{2}+i \beta\right)^{1 / 2}}{\phi+\left(\phi^{2}-\phi_{c}^{2}+i \beta\right)^{1 / 2}}\right|^{2},
$$

with $\phi$ defined as in the inset of Fig. 1 and a critical angle $\phi_{c}=0.222^{\circ}$ for a silicon substrate and $\lambda=1.5405 \AA$ radia- 


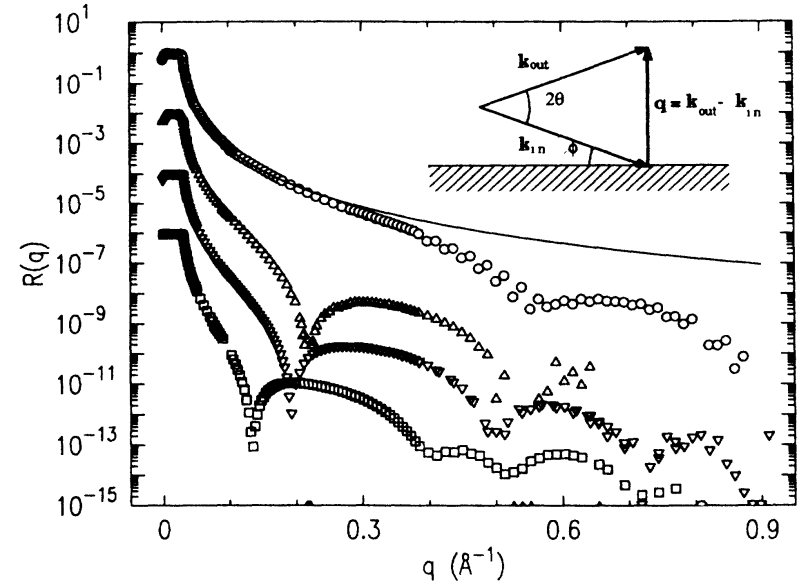

FIG. 1. Normalized reflectivity data from several samples. Successive data sets are displaced by 100 times and error bars omitted for clarity. (_-) Theoretical reflectivity from an ideal step interface with bulk silicon density. ( $O$ ) Uncoated silicon sample in helium; the "pairing" of points occurs for two scans taken $60 \mathrm{~min}$ apart and is probably due to the build up of contaminants on the surface. $(\triangle)$ 10-carbon chain alkylsiloxane. $(\nabla)$ 12-carbon chain alkylsiloxane. ( $\square$ ) 18-carbon chain alkylsiloxane. The inset shows a schematic diagram of the scattering vectors for the specular reflectivity condition, where $2(\phi)=2 \theta$.

tion. Equation (1) can be reexpressed in terms of the scattering vector $q=(4 \pi / \lambda) \sin (\phi)$ (Fig. 1) as

$$
R_{F}(q)=\left|\frac{q-\left(q^{2}-q_{c}^{2}+2 i / \mu\right)^{1 / 2}}{q+\left(q^{2}-q_{c}^{2}+2 i / \mu\right)^{1 / 2}}\right|^{2},
$$

where $q_{c} \approx(4 \pi / \lambda) \sin \left(\phi_{c}\right)$ is the "critical wave vector" in air and is independent of the wavelength $q_{c}=0.0316 \AA^{-1}$ for silicon. This form for the reflectivity, shown for silicon as the solid line in Fig. 1, includes a slight rounding near the critical wave vector due to the small absorption factor. Away from $q \approx q_{c}$ absorption effects are negligible. For $q<q_{c}$ the radical is almost pure imaginary and the reflectivity is essentially $100 \%$ (i.e., total external reflection). Well above the critical angle the reflectivity is given by $R_{F}(q) \approx\left(q_{c} / 2 q\right)^{4}$.

For real surfaces the reflectivity can be expressed in terms of the average electron density ${ }^{12,19,37}$

$$
\begin{aligned}
& R(q)=R_{F}(q)|\Phi(q)|^{2}, \\
& \Phi(q)=\int \frac{1}{\rho_{\infty}}\left\langle\frac{d \rho}{d z}\right\rangle e^{i q z} d z,
\end{aligned}
$$

where $\langle d \rho / d z\rangle$ is the derivative of the electron-density profile averaged over the in-plane coherence length of the $x$ rays and $\rho_{\infty}$ is the electron density of the semi-infinite bulk. This form is valid for angles greater than approximately twice the critical angle, where refraction effects are negligible (i.e., when the Born approximation for the scattering is valid).

It is convenient to model the in-plane averaged elec- tron density of a simple surface by a Gaussian smeared step from $\rho=0$ to $\rho=\rho_{\infty}:{ }^{12}$

$$
\langle\rho(z)\rangle=\frac{\rho_{\infty}}{2}\left[1+\operatorname{erf}\left[\frac{z}{2 \sigma}\right]\right] .
$$

The average normal derivative is given by the Gaussian form

$$
\left\langle\frac{d \rho}{d z}\right\rangle=\rho_{\infty} \frac{1}{\left(2 \pi \sigma^{2}\right)^{1 / 2}} e^{-z^{2} / 2 \sigma^{2}}
$$

where $\sigma$, the root-mean-square average of the surface width, results from both the intrinsic width of the interface and the mean-square average of the roughness of the surface. ${ }^{19}$ The Fourier transform described by Eq. (4) yields

$$
R(q)=R_{F}(q) e^{-\sigma^{2} q^{2}},
$$

an expression reminiscent of the Debye-Waller factor for solids. For $q \leq 0.4 \AA^{-1}$ the deviations between the measured reflectivity for the "bare silicon-silicon-oxide" wafer and the Fresnel reflection law in Fig. 1 are well described by a model surface of the form of Eq. (5) with an interface width of approximately $2.8 \AA$. This model does not explain either the reflectivity of the uncoated silicon for $q>0.4 \AA^{-1}$ nor the reflectivity from the alkylsiloxane coated samples that are also shown in Fig. 1. As implied above, since the reflectivity predicted by this model falls off with increasing incident angles as the product of a Gaussian and the $1 / q^{4}$ term, the intensity becomes the limiting factor in measuring the reflectivity at larger angles.

The simplest physically reasonable model for the surface of the siloxane coated surface consists of a silicon substrate with electron density $\rho_{\mathrm{Si}}$ that is covered uniformly with a hydrocarbon layer of length $L$ and electron density $\rho_{\mathrm{CH}}$. If the silicon-alkane and alkane-air interfaces have widths $\sigma_{1}$ and $\sigma_{2}$, respectively, the normal derivative is of the form

$$
\begin{aligned}
\left\langle\frac{d \rho}{d z}\right\rangle= & \left(\rho_{\mathrm{Si}}-\rho_{\mathrm{CH}}\right) \frac{1}{\left(2 \pi \sigma_{1}^{2}\right)^{1 / 2}} e^{-z^{2} / 2 \sigma_{1}^{2}} \\
& +\rho_{\mathrm{CH}} \frac{1}{\left(2 \pi \sigma_{2}^{2}\right)^{1 / 2}} e^{-(z-L)^{2} / 2 \sigma_{2}^{2}} .
\end{aligned}
$$

Application of Eqs. (3) and (4) to Eq. (8) generates

$$
\begin{aligned}
\frac{R(q)}{R_{F}(q)}=|\Phi(q)|^{2}= & \frac{\left(\rho_{\mathrm{Si}}-\rho_{\mathrm{CH}}\right)}{\rho_{\mathrm{Si}}} e^{-q^{2} \sigma_{1}^{2} / 2} \\
& +\left.\frac{\rho_{\mathrm{CH}}}{\rho_{\mathrm{Si}}} e^{-q^{2} \sigma_{2}^{2} / 2} e^{-i q L}\right|^{2} .
\end{aligned}
$$

For small angles, such that $q \sigma_{1,2} \ll 1$, this expression simplifies to the form

$$
\begin{aligned}
\frac{R(q)}{R_{F}(q)} \approx & {\left[\frac{\rho_{\mathrm{Si}}-\rho_{\mathrm{CH}}}{\rho_{\mathrm{Si}}}\right]^{2}+\left(\frac{\rho_{\mathrm{CH}}}{\rho_{\mathrm{Si}}}\right)^{2} } \\
& +2\left[\frac{\rho_{\mathrm{Si}}-\rho_{\mathrm{CH}}}{\rho_{\mathrm{Si}}}\right]\left[\frac{\rho_{\mathrm{CH}}}{\rho_{\mathrm{Si}}}\right] \cos (q L) .
\end{aligned}
$$


Since $\left(\rho_{\mathrm{Si}}-\rho_{\mathrm{CH}}\right) / \rho_{\mathrm{Si}} \approx \rho_{\mathrm{CH}} / \rho_{\mathrm{Si}}$, this model predicts the observed minimum in the reflectivity (Fig. 1) when $q=(4 \pi / \lambda) \sin (\phi)=\pi / L$, where $L$ is approximately equal to the thickness of hydrocarbon film. On the other hand, this model does not explain the structure in the reflectivity at $q>0.3 \AA^{-1}$ that can be seen in Fig. 1 .

A more general model, with $N$ separate layers, has the form

$$
\left\langle\frac{d \rho}{d z}\right\rangle=\sum_{0}^{N}\left(\rho_{i}-\rho_{i+1}\right) \frac{1}{\left(2 \pi \sigma_{i+1}^{2}\right)^{1 / 2}} e^{-\left(z-D_{t}\right)^{2} / 2 \sigma_{i+1}^{2}},
$$

where $\rho_{0}$ corresponds to the electron density of the substrate $\left[\rho_{\infty}\right.$ of Eq. (5)], which in the present example is crystalline silicon, $\rho_{N+1} \approx 0$ is the density in air, $L_{i}$ is the thickness of the $i$ th layer and $D_{i}=\sum_{j=1}^{i} L_{j}$ is the distance from the crystalline silicon surface to the interface between the $i$ th and the $(i+1)$ st layers (i.e., $\left.D_{0}=0\right)$. The Fourier transform for this form yields

$$
\begin{aligned}
\frac{R(q)}{R_{F}(q)} & =|\Phi(q)|^{2} \\
& =\left|\sum_{0}^{N}\left[\frac{\rho_{i}-\rho_{i+1}}{\rho_{0}}\right) e^{-i q D_{i}} e^{-q^{2} \sigma_{i+1}^{2} / 2}\right|^{2},
\end{aligned}
$$

where $\rho_{0}=\rho_{\mathrm{Si}}$ is the density of crystalline silicon. We will show below that the data for the alkylsiloxane coated silicon wafers shown in Fig. 1 are well described by a model in which $N=3$.

The coherence length for the $\mathrm{x}$ rays is a function of the spectrometer resolution, this being a function of the slit widths and $x$-ray path lengths. Typically the coherence length in the plane of the surface is also a function of the inverse incident angle. At the rotating anode, this length is of the order of $4 \times 10^{4} \AA$ at the critical angle and $3 \times 10^{3} \AA$ at $3^{\circ}$. The corresponding lengths at the synchrotron are about $10^{5}$ and $8 \times 10^{3} \AA$.

Surfaces that are inhomogeneous in the plane of the surface give rise to nonspecular surface diffuse scattering (SDS). Although SDS has been observed by us and others, ${ }^{19,38,39}$ for the silicon substrates used in this study the surface diffuse scattering integrated over the spectrometer resolution at $q \approx 0.04 \AA^{-1}$ was less than $\approx 10^{-2}$ of the intensity of the specular reflection, and we have not carried out systematic measurements of surface diffuse scattering from these samples.

\section{EXPERIMENTAL DETAILS}

\section{Preparation of samples (Ref. 34)}

Sample substrates were made from highly polished silicon (100) wafers obtained from Semiconductor Processing Corporation of Boston, Massachusetts. Each sample consisted of a 1-in strip cut from a 3-in-diam wafer that was either $0.08,0.125$, or 0.200 in thick. The thinnest 0.08 -in wafers were found to be warped with typical surface normal variations of about $0.05^{\circ}$ over the central 5 $\mathrm{cm}$ of the wafer, compared to $0.005^{\circ}$ for the corresponding region of the thicker wafers. Although early studies and some of the synchrotron data were taken on the thin substrates, most of the data reported were carried out on the 0.125 -in wafers.

The silicon wafers were cleaned by immersing them in an $\mathrm{H}_{2} \mathrm{O}_{2}+$ sulfuric acid mixture $(70 \mathrm{vol}$. \% concentrated $\mathrm{H}_{2} \mathrm{SO}_{4}, 30$ vol. $\% \mathrm{H}_{2} \mathrm{O}_{2}$ at $90^{\circ} \mathrm{C}$ for $30 \mathrm{~min}$ ). This strongly oxidizing combination removes all organic contaminants on the surface, but does not disturb the native silicon oxide layer. The wafers were then rinsed and stored under distilled water before use. Prior to preparing the monolayers, the wafers were removed from the water and blown dry under a stream of argon.

Decyl-, undecyl-, dodecyl-, tetradecyl-, hexadecyl-, and octadecyltrichlorosilane [i.e., $\mathrm{Cl}_{3} \mathrm{Si}-\left(\mathrm{CH}_{2}\right)_{n}-\mathrm{CH}_{3}$ with $n=9,10,11,13,15,17$, respectively] were used to form the alkane monolayers. Each wafer was allowed to react with a solution of alkyl trichlorosilane $(\approx 0.5 \mathrm{wt}$. \% in hexadecane) for up to $24 \mathrm{~h}$ before being removed from the solution and rinsed with hexane and ethanol. These operations were performed under a dry, inert atmosphere when conditions of high ambient humidity existed. The samples were autophobic to solution on removal from the solution of alkyl trichlorosilane.

The following procedure was followed to minimize surface contamination. Ellipsometric measurements of the monolayer thicknesses were made within $5 \mathrm{~min}$ of removal of the sample from water. ${ }^{34}$ Coated wafers were typically then stored in air for periods as long as one week before x-ray measurements were made. No change was noted in the $x$-ray data between measurements of fresh samples and of samples stored for up to one month after preparation. Immediately prior to taking the x-ray data, samples were rinsed with dry ethanol to remove organic contamination, blown dry with dry nitrogen, and immediately transferred to the $\mathrm{x}$-ray spectrometer. X-ray photoelectron spectroscopy (XPS) data for the samples were taken some time (generally about two months) after the $x$-ray measurement.

Partially complete monolayers were formed by removing the sample from the solution in a time shorter than that required for a full film to form. ${ }^{40}$ Ellipsometric measurements were used to obtain one estimate of the degree of coverage. ${ }^{34}$ The alkene terminated film was made by the same method as for the alkane films, but starting with a trichlorosilane with the appropriate alkene tail, namely $\mathrm{Cl}_{3} \mathrm{Si}-\left(\mathrm{CH}_{2}\right)_{15}-\mathrm{CH}=\mathrm{CH}_{2}$. The brominated sample was made from one of the alkene terminated samples, after the initial $x$-ray reflection measurement was completed, by immersing the sample in a $2 \%$ by volume solution of elemental bromine in methylene chloride. The fluorocarbon sample was formed in a similar manner to the alkylsiloxane monolayers, again using the relevant precursor $\left(\mathrm{Cl}_{3} \mathrm{Si}-\left(\mathrm{CH}_{2}\right)_{2}-\left(\mathrm{CF}_{2}\right)_{7}-\mathrm{CF}_{3}\right)$. The use of this form was necessitated by chemical restrictions which make the much simpler fluorosilane molecule $\mathrm{Cl}_{3} \mathrm{Si}-$ $\left(\mathrm{CF}_{2}\right)_{9}-\mathrm{CF}_{3}$ difficult to synthesize. Further details of the sample preparations are given in other papers. ${ }^{34,40}$

\section{$\mathrm{X}$-ray technique}

Most of the data reported here were taken on beam line X-22B at the National Synchrotron Light Source 
(NSLS) facility at Brookhaven National Laboratory. Some of the low-angle data were taken on the rotating anode X-ray generator of the Harvard Materials Research Laboratory in order to locate the position of the lowestorder destructive interference minimum and to make preliminary judgements of sample quality.

The rotating anode measurements were made using the configuration shown in Fig. 2(a). The monochromator was either a single- or triple-bounce germanium (111) crystal [Ge(111)] set to accept copper $K \alpha_{1}$ radiation (wavelength $1.5405 \AA$ ). At small incident angles $\phi$, the intersection of a collimated beam of width $w$ covers a length $\sim w / \sin (\phi) \gg>w$, with the size of the beam incident on the sample being defined by slit $S_{2}$ and some preliminary collimation provided by slit $S_{1}$. For angles below $\approx 1^{\circ}$ the dimensions of $S_{2}$ were approximately 100 $\mu \mathrm{m}$ horizontal width by $6 \mathrm{~mm}$, and $500 \mu \mathrm{m} \times 6 \mathrm{~mm}$ for larger angles, with similar dimensions for $S_{1}$. These slit dimensions were chosen to satisfy the conditions of (1) all the beam being incident on the central $50 \mathrm{~mm}$ of the sample, (2) avoiding detector saturation, and (3) maximizing the incident flux at large incident angles. The principal purpose of slit $S_{3}$ was to reduce the background scattering by trimming the tails of the slit scattering from $S_{2}$. $S_{3}$ was closed symmetrically to the point that it had a measurable effect on the count rate and was then opened slightly. $S_{4}$ was opened to dimensions of approximately $1 \times 10 \mathrm{~mm}^{2}$ assuring that all the beam reflected off the

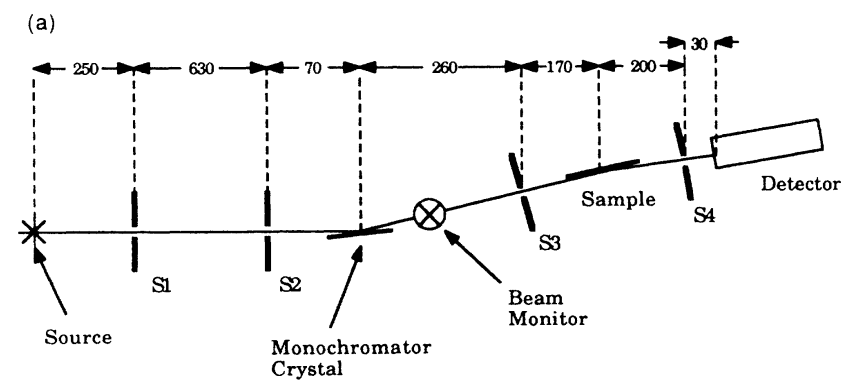

(b)

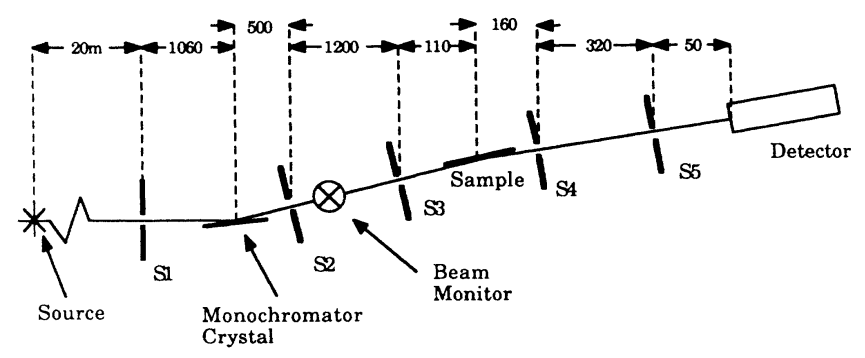

FIG. 2. (a) Schematic of the rotating anode configuration. $S_{2}$ was the beam-defining slit; the monochromator was either triple-bounce or single-bounce germanium. All lengths are in millimeters, with typical slit dimensions given in the text. (b) Schematic of the synchrotron configuration (beamline X-22B at NSLS). $S_{1}$ defined the coarse horizontal and vertical beam, fine beam definition being obtained from slit $S_{2}$; the monochromator was a single-bounce germanium crystal. sample was detected. The monitor and detector were $\mathrm{NaI}(\mathrm{Tl})$ scintillation counters, the monitor being placed at $90^{\circ}$ to the beam with a small piece of plastic scattering approximately $0.03 \%$ of the beam into the detector. A reflection intensity dynamic range of about $10^{7}$ was achieved for a typical series of scans lasting approximately $12 \mathrm{~h}$.

At the synchrotron a wavelength of $1.7096 \AA$ was used the experimental configuration being shown in Fig. 2(b). A single bounce Ge(111) crystal was used. Slit $S_{1}$ actually consisted of two slits about $50 \mathrm{~mm}$ apart: the first was a triangular slit used to coarsely define the useful part of the beam in the horizontal direction, the second slit defining the vertical definition of the beam. Although these slits were crudely set, they significantly reduced the background scattering inside the experimental hutch. $\mathrm{S}_{2}$ was the beam defining slit, with slit $S_{3}$ trimming the tails of the slit scattering but not affecting the counts in the main beam. The slit widths were similar to those used at Harvard except that, at the largest angles, a beam width of $1 \mathrm{~mm}$ was used. Because of the very intense beam at very small angles (below $1^{\circ}$ ), the detector was placed at $90^{\circ}$ to the beam with a small sheet of plastic scattering about $0.03 \%$ of the beam into the detector. The detector was switched to a direct position at about $0.8^{\circ} . S_{3}$ was opened to $1 \mathrm{~mm}$ at an angle of about $3^{\circ}$. Slit $S_{4}$ was set to cut down the direct scattering from sources other than the sample, and $S_{5}$ was set wide enough to accept all the specular beam reflected from the sample. All data were normalized to the counts recorded in a beam monitor located between the beam defining slit $S_{2}$ and the trimming slit $S_{3}$. It consisted of a second plastic sheet that scattered about $0.02 \%$ of the direct beam into a second scintillation detector at $90^{\circ}$ to the direct beam. A reflected intensity dynamic range of $10^{9}$ was obtained over the period of about $4 \mathrm{~h}$ necessary to record a typical set of scans for one sample.

For both experimental configurations samples were aligned by using the diffractometer in a nondispersive three-crystal mode in which a single- or triple-bounce $\mathrm{Ge}(111)$ analyzer crystal was placed between the last slit and the detector. With the sample removed the analyzer was in a dispersive orientation; nevertheless, a good measure of the incident angle for the direct beam was obtained by rotating the analyzer crystal to maximize the signal in the detector. In order to obtain an approximate alignment, the sample was then translated into the beam and, by an iterative process in which $\phi$ was rotated and the sample translated, the sample was aligned parallel to and obscuring half of the beam. Next the sample was rotated to an angle just below the critical angle (typically $0.15^{\circ}$ ) and the detector was scanned through the specular reflection (i.e., a $2 \theta$ scan) in order to check the alignment and figure error of the sample. Since the initial alignment procedure was prone to errors arising from macroscopic substrate warping (typically from the edges from which no scattering is measured), the final sample alignment was obtained by setting $\phi$ equal to half of $2 \theta$. The analyzer crystal was then removed and the detector centered on the specularly reflected beam passing through slit $S_{5}$. Finally, the sample was translated through the 

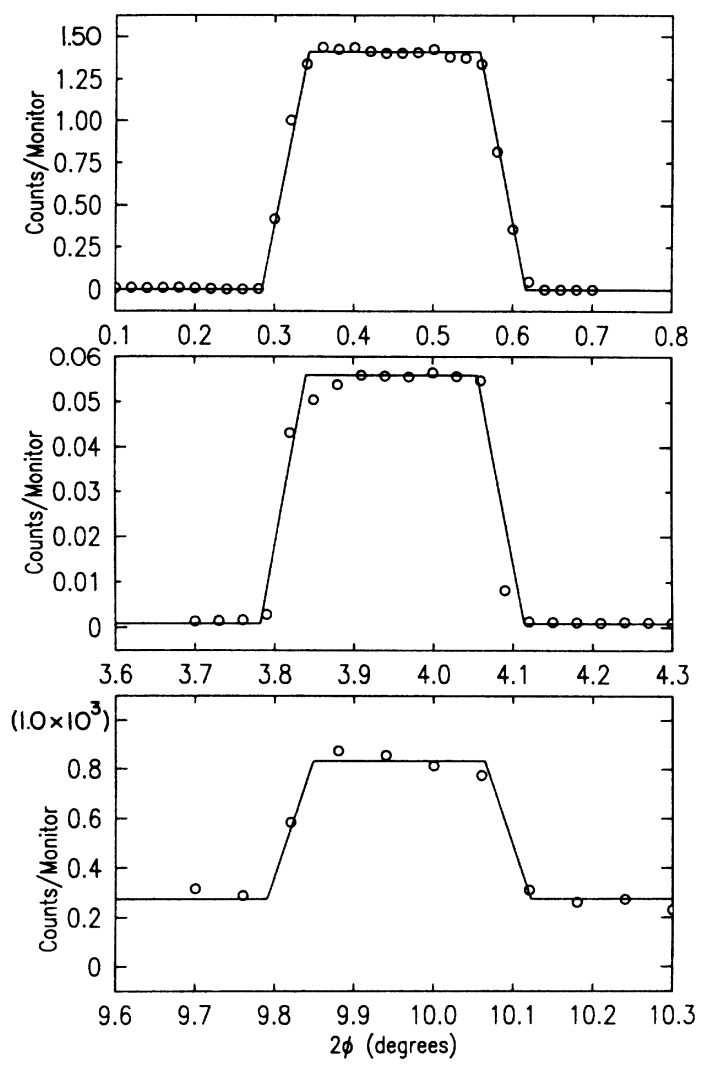

FIG. 3. Three typical beam profiles obtained by scanning $2 \theta$ at fixed $\phi$ for the spectrometer shown in Fig. 2(b). The trapezoidal shape is a function of the resolution of the $S_{4}$ slit and the beam profile, the former being much larger to ensure all of the reflected beam enters the detector. The lines give the best fit for the amplitude of a trapezoid whose shape was fixed and determined by the incoming beam dimensions and the detector slit width. For all specular scans the detector was positioned in the center of the trapezoid.

beam parallel to the surface normal in order to ensure that the incident beam was correctly centered on the sample.

Although the angular dependence of the specular reflectivity was measured by a series of " $\phi-2 \theta$ " scans in which $\phi$ is equal to half of $2 \theta$, the alignment was frequently checked by performing $2 \theta$ scans at fixed $\phi$. This procedure ensured accurate sample alignment to within $0.01^{\circ}$ and was also a check that the figure error of a sample was acceptable. Since the nonspecular diffuse scattering depended on the incident angle $\phi$, "background scattering" was subtracted from the signal $I(\phi, 2 \theta)_{\phi=\theta}$ observed in the specular condition, i.e., specular reflection

$$
R(\phi)=I(\phi, 2 \phi)-\frac{1}{2}\left[I\left(\phi, 2 \phi-0.3^{\circ}\right)+I\left(\phi, 2 \phi+0.3^{\circ}\right)\right] .
$$

The background for three different angles of incidence are illustrated for data taken at NSLS in Fig. 3 by $\phi$ scans at fixed $2 \theta$. The signal reported as the specular reflectivity is obtained by subtracting the background count rate from the peak count rate, as described.
Although the experiments were carried out with the samples contained in a sealed cell that was filled with air or helium, the results discussed below demonstrated that airborne organic materials were not fully eliminated from the helium-filled cell. The $x$ rays were incident through Kapton windows, with an angular access of up to $7^{\circ}$ and approximately $75 \%$ transmissivity.

\section{RESULTS}

\section{Alkylsiloxanes with $\mathrm{C10}, \mathrm{C12}$, and $\mathrm{C18}$ alkyl chains}

\section{Data and simple interpretations}

Figure 1 shows the results of synchrotron measurements of the reflectivity $R(q)$ (after background subtraction) for alkylsiloxane monolayers of differing length and for the uncoated silicon. This figure also shows the Fresnel reflectivity for an ideal step surface of a material with the bulk density of silicon. Without any sophisticated analysis, there are a number of prominent features that can be immediately interpreted. The reflectivity of all of the alkylsiloxane-covered samples exhibit structure, most notably a sharp minimum at a scattering vector of between 0.1 and $0.25 \AA^{-1}$, and other minima and maxima at larger $q$.

The most obvious interpretation is that the first minimum is the result of destructive interference between reflections from the front and back surfaces of the alkane layer of thickness $L$. For thin enough films, or for films in which the electron density is not too high, the position of the minimum in this interpretation is given by the condition that $q L=\pi$ or $\phi=\sin ^{-1}(\lambda / 4 L)$. For either thicker or denser films, refraction effects are, however, important and the destructive interference occurs for $q^{\prime} L$ $=L\left(q^{2}-q_{c}^{2}\right)^{1 / 2}=\pi$. For a fully formed hydrocarbon layer of the type of interest here, $q_{c} \approx 0.021 \AA^{-1}$ would be the critical wave vector of a semi-infinite sample with the same electron density as the hydrocarbon layer. The positions of the minima for the $\mathrm{C} 10, \mathrm{C} 12$, and C18 correspond to $q=0.21$ and 0.19 , and $0.13 \AA^{-1}$, respectively. Taking the refracton correction into account, the thicknesses of the $\mathrm{C} 10, \mathrm{C} 12$, and $\mathrm{C} 18$ alkane layers, for this interpretation of the position of minima, correspond to $14.4,16.3$, and $23.6 \AA$, respectively. These values for the thickness $L$ of the alkane layers should be compared with the published expression $L=1.265 n+1.5 \AA$, which gives $14.2,16.7$, and $24.3 \AA$ for the maximum extension of an aliphatic chain $-\left(\mathrm{CH}_{2}\right)_{n-1} \mathrm{CH}_{3}$ with $n=10,12$, and 18 respectively. ${ }^{41}$

A similarity of the reflectivities from these three samples is that they all fall below the Fresnel curve. This indicates that the reflecting interfaces are not ideally sharp, but have some associated widths. The deep nature of the minima (cancellation of between $10^{-2}$ and $10^{-3}$ ) indicates that at the angle of the minimum, the amplitude of the wave reflected from the top and bottom interfaces of the hydrocarbon chain are of almost equal magnitude. Since the known ratio of the electron density of bulk hydrocarbon to that of silicon is approximately 0.38 , the expected ratio of minima to maxima would be approximately 
$[(1.2) \times 0.38]^{2} \approx 0.06$ if interfacial widths were neglected. The fact that this prediction is approximately an order of magnitude larger than the observed ratio implies that the different interfaces have different widths. Closer examination of the reflectivity also reveals that minima at larger angles do not occur at positions that are integral multiples of the positions of the smallest-angle minimum. All of these features can be understood in terms of a more complex three-layer model that will be described in detail below.

\section{Uncoated silicon sample}

Evidence of an experimental problem with surface contamination of the uncoated silicon sample during the $\mathrm{x}$ ray measurements can be seen in the data in Fig. 1. At large scattering vectors, alternate points were measured in scans taken approximately $60 \mathrm{~min}$ apart and, as can be seen, the points from the two different scans are offset from one another. We believe this is due to the continuous build up of a contamination layer on the sample. At the time of the measurement this layer was probably about $5 \AA$ thick; however, the progressive shifting of the minimum to lower angles in data taken a few hours later confirmed the build up of contaminants on the surface. Some of the contamination is probably caused by the presence of organic materials or water in the helium flowing through the sample cell during the experiment since there was a significantly slower build up on samples left exposed to air for a similar length of time. No such problem was observed with the lower-energy alkylsiloxanecoated surfaces.

\section{Detailed analysis}

Detailed analysis of the alkylsiloxane surface electron density was carried out by least-squares fitting of the data to a version of the $N$-layer model for $\Phi(q)$ that was corrected for the effects of refraction. The correction involved replacing the $\left\{q D_{i}\right\}$ in the factor

$$
\left\{e^{-i q D_{i}} e^{-q^{2} \sigma_{t+1}^{2} / 2}\right\}
$$

of Eq. (12) by

$$
q D_{i}=\sum_{j=1}^{i} q_{j} L_{j},
$$

where $q_{j}=\left(q^{2}-q_{c j}^{2}\right)^{1 / 2}$ and $q_{c j}$ is the critical wave vector for the $j$ th layer. In principle, a similar correction is required for the Gaussian term. The corrections are, however, small and were neglected.

\section{C18 alkyl chain monolayer}

Figure 4 shows the data for the C18-coated silicon wafer in the form of $R(q) / R_{F}(q)$. The solid lines display a set of fits for models with 1, 2, and 3 layers, respectively, i.e., $N=1,2$, and 3 in Eq. (12). The parameters of these fits are given in the columns labeled $N=1,2$, and $3^{(+)}$in Table I. The $N=1$ and 2 models are obviously inadequate. In addition, for the $N=2$ model the fitting algorithm was unable to fix either the width of the

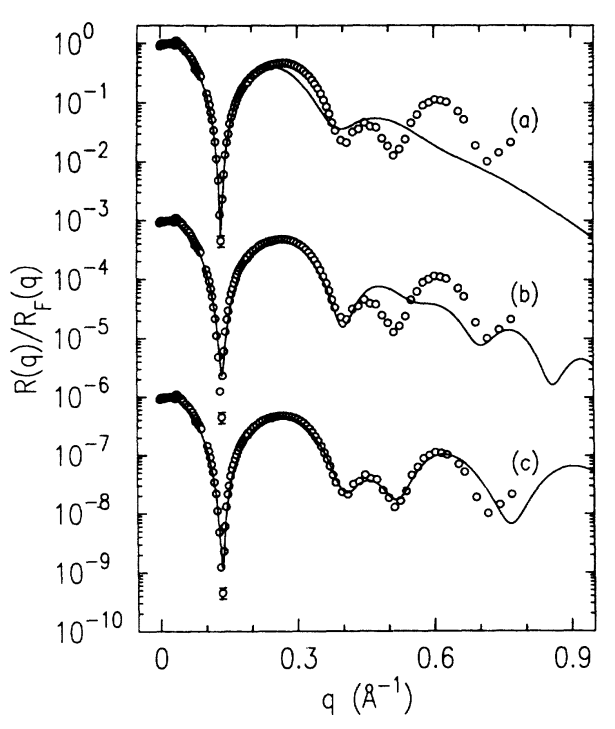

FIG. 4. Reflectivity and analysis of an alkylsiloxane monolayer containing an 18-carbon chain using one-, two-, and three-layer models. The data are shown after normalization to the silicon Fresnel reflectivity, and hence the $y$ axis represents $|\Phi|^{2}$. (a) shows the one-layer model fit to the data at low $q$. This model accurately fitting only the first minimum; the twolayer fit (b) is quite accurate out to about $0.45 \AA^{-1}$ and qualitatively predicts the peak and dip positions at larger angles. The three-layer model (c) reasonably fits the data over the entire range. The best fit parameters for the different models are given in Table $I$ in the $N=1,2$, and $3^{(+)}$columns.

silicon-silicon-oxide interface $\left(\sigma_{01}\right)$ or the thickness of the silicon oxide layer $\left(L_{1}\right)$. The values of these parameters appearing in Table $\mathrm{I}$ for $N=2$ were chosen such that the maxima and minima in the model were at approximately the same positions as in the data, and the depth of the second minimum was also approximately correct. Given the obvious inadequacies, confidence limits for the parameters of these models are not particularly meaningful.

The motivation for the layer $L_{1}$ comes from the wellknown fact that on exposure to $\mathrm{O}_{2}$, crystalline silicon forms a relatively stable oxide layer that is about $10-20$ $\AA$ thick. ${ }^{42}$ In addition, it is difficult to see how a hydrocarbon layer on its own could give rise to the nonintegral positions of the high-angle minima. A more compelling case specific to the present data are the results for the Patterson function (Fig. 5)

$$
\begin{aligned}
Z(s) & \equiv \frac{1}{2 \pi} \int_{-\infty}^{\infty} d q|\Phi(q)|^{2} e^{-i q s} \\
& =\frac{1}{\rho_{\infty}^{2}} \int_{-\infty}^{\infty}\left\langle\frac{d \rho(z)}{d z}\right\rangle\left\langle\frac{d \rho(s+z)}{d z}\right\rangle d z
\end{aligned}
$$

calculated from the data for $R(q) / R_{F}(q)=|\Phi(q)|^{2}$ in Fig. 4. The solid line in Fig. 5 was calculated directly from the data by correcting the critical angle to cor- 
TABLE I. Parameters for fits of the $N=1,2$, and 3 layer model described by Eq. (12) to the data for the C18 alkylsiloxane-coated silicon wafer. The results calculated from the columns $N=1,2$, and $3^{(+)}$are shown in Fig. 4 . The model reflectivities for parameters $3^{(+)}, 3^{(+1)}, 3^{(+2)}$, and $3^{(-)}$are illustrated in Figs. $7(\mathrm{a}), 7(\mathrm{~b}), 7(\mathrm{c})$, and $7(\mathrm{~d})$, respectively, and the real-space densities are illustrated in Fig. 8. The notation $3^{( \pm)}$distinguishes between models in which $\rho_{2}(\gtrless) \rho_{0}$. The $N=1,2,3^{(+)}$, and $3^{(-)}$columns are best fits, while the $N=3^{(+1)}$ and $3^{(+2)}$ columns indicate the boundaries of acceptable fits $\left(\chi^{2} \approx 1.25 \chi^{2}\right.$ minimum). The sixth line gives the lengths as measured by the graphical technique described in the text.

\begin{tabular}{|c|c|c|c|c|c|c|c|}
\hline Layer & & $N=1$ & $N=2$ & $3^{(+)}$ & $3^{(+1)}$ & $3^{(+2)}$ & $3^{(-)}$ \\
\hline \multicolumn{8}{|c|}{ Layer thickness $L,(\AA)$} \\
\hline $\mathrm{SiO}_{2}$ & $L_{1}(\stackrel{\AA}{\AA})$ & & $17.4^{\mathrm{a}}$ & $16.8 \pm 0.9$ & 17.5 & 16.7 & $12.7 \pm 1.5$ \\
\hline Interface & $L_{2}(\AA)$ & & & $0.7 \pm 0.6$ & 0.014 & 0.8 & $7.0 \pm 1.5$ \\
\hline$-\left(\mathrm{CH}_{2}\right)-$ & $L_{3}(\AA)$ & 23.7 & 23.0 & $23.5 \pm 0.3$ & 23.4 & 23.5 & $21.3 \pm 0.5$ \\
\hline$-\left(\mathrm{CH}_{2}\right)-$ graph & $L_{3}(\AA)$ & 20.8 & 20.4 & $21.4 \pm 0.5$ & 21.2 & 21.6 & $21.2 \pm 0.5$ \\
\hline \multicolumn{8}{|c|}{$\rho_{J} / \rho_{0}$} \\
\hline $\mathrm{SiO} \rho_{1} / \rho_{0}$ & & & 0.968 & $0.96 \pm 0.01$ & 0.96 & 0.96 & $0.96 \pm 0.01$ \\
\hline Interface & $\rho_{2} / \rho_{0}$ & & & $1.25_{-0.1}^{+20}$ & $20^{\mathrm{a}}$ & $1.12^{\mathrm{a}}$ & $0.82 \pm 0.15$ \\
\hline$-\left(\mathrm{CH}_{2}\right)-$ & $\rho_{3} / \rho_{0}$ & $0.45^{\mathrm{a}}$ & 0.42 & $0.43_{-0.02}^{+0.05}$ & 0.46 & 0.43 & $0.43_{-0.02}^{+0.06}$ \\
\hline \multicolumn{8}{|c|}{$\sigma_{\imath \jmath}(\AA)$} \\
\hline $\mathrm{Si} / \mathrm{SiO}_{2}$ & $\sigma_{01}\left(\AA_{0}^{\AA}\right)$ & & $1.0^{\mathrm{a}}$ & $1.0^{\mathrm{a}}<2.0$ & $1^{\mathrm{a}}$ & $1^{\mathrm{a}}$ & $1^{\mathrm{a}}<2.0$ \\
\hline $\mathrm{SiO}_{2} /$ Inter. & $\sigma_{12}\left(\AA \AA_{0}\right)$ & & & $1.0^{\mathrm{a}}<3.0$ & 2.39 & 0 & $1^{\mathrm{a}}<2.0$ \\
\hline Inter. $/\left(\mathrm{CH}_{2}\right)$ & $\sigma_{23}\left(\AA_{0}\right)$ & 4.9 & 4.2 & $3.2 \pm 0.5$ & 2.44 & 3.4 & $2.4 \pm 0.8$ \\
\hline$\left(\mathrm{CH}_{2}\right) / \mathrm{Air}$ & $\sigma_{34}(\AA)$ & $2.6^{\mathrm{a}}$ & $2.3^{\mathrm{a}}$ & $2.4 \pm 0.3$ & 2.6 & 2.3 & $2.4 \pm 0.4$ \\
\hline
\end{tabular}

aParameter held constant during fit.

respond to that inside the bulk, assuming $|\Phi(q)|^{2}=|\Phi(-q)|^{2}$ and using a smooth Gaussian to extrapolate from the last measured point at $q \approx 0.8 \AA^{-1}$ to the vanishing of $|\Phi(q)|^{2}$ at $q= \pm 8 \AA^{-1}$ (well beyond the measured range, where the reflectivity is essentially zero).

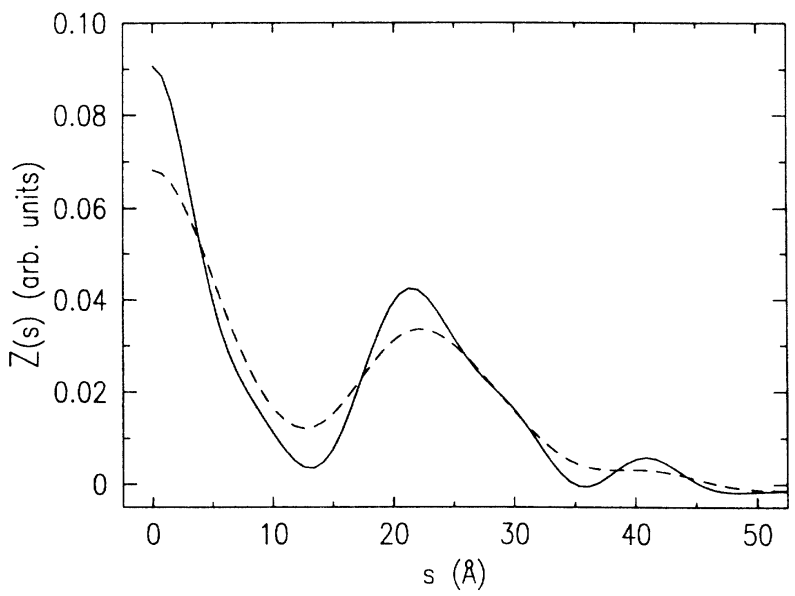

FIG. 5. Patterson function calculated from the data in Fig. 4 for the 18-carbon chain alkylsiloxane monolayer. The solid curve was calculated directly from the Fourier transform of the data; the dashed curve was obtained by multiplying the reflectivity data by a Gaussian of the form $\exp \left(-q^{2} / 2 \sigma_{P}^{2}\right)$, where $\sigma_{P}=0.3 \AA^{-1}$, prior to taking the Fourier transformation. This is equivalent to convoluting the Patterson function with a Gaussian of the form $\sim \exp \left[-\left(z-z^{\prime}\right)^{2} \sigma_{P}^{2} / 2\right]$. The fact that the peak at about $40 \AA$ survives the convolution process indicates that it is a real feature of the data and not an artifact of the data termination.
For the C18 alkylsiloxane this Gaussian corresponded to a surface having a $3.6-\AA$ interfacial width. The peak at $s \approx 40 \AA$ in Fig. 5 indicates that, in addition to the main hydrocarbon layer of approximately $20 \AA$ thickness, there is a second layer, also of about $20 \AA$ thickness, with an interface that is either $20 \AA$ above the alkane-air interface and $40 \AA$ above the silicon-oxide-alkane interface, or $20 \AA$ below the silicon-oxide-alkane interface and $40 \AA$ below the alkane-air interface. While the former suggestion is unphysical, the latter could correspond to the native silicon oxide layer, the silicon-silicon-oxide interface convoluted with the hydrocarbon-air interface being responsible for the peak at about $40 \AA$. The values of the parameters in Table I for the $N=2$ model with the electron density $\rho_{1}=0.968$ and a layer thickness $L_{1}=17.4 \AA$ are consistent with the presence of a silicon oxide layer.

The most important uncertainty associated with the Patterson function is whether or not the structure could be an artifact associated with the way the data are extrapolated past the last measured point. ${ }^{43}$ The dashed line indicates the result that is obtained from multiplying the data by a Gaussian with $\sigma_{P}=0.3 \AA^{-1}$ such that the data for $q$ greater than $0.8 \AA^{-1}$ make no important contribution to the integral, i.e., $\exp \left[-(0.8 / 0.3)^{2} / 2\right] \approx 3 \times 10^{-4}$. The Patterson function calculated is equivalent to viewing the autocorrelation function through a "real-space Gaussian filter" which reduces the amplitudes of the peaks and increases their widths, e.g.,

$$
\begin{aligned}
Z_{G}(s) & \equiv \frac{1}{2 \pi} \int_{-\infty}^{\infty} d q|\Phi(q)|^{2} e^{-q^{2} / 2 \sigma_{P}^{2}} e^{-i q s} \\
& =\int_{-\infty}^{\infty} Z\left(s+s^{\prime}\right) \frac{1}{\left(2 \pi \sigma_{P}^{2}\right)^{1 / 2}} e^{\left[-\left(s^{\prime}\right)^{2} \sigma_{P}^{2} / 2\right]} d s^{\prime} .
\end{aligned}
$$


Since the structure at $s \approx 40 \AA$ is still present (albeit smeared out) it cannot be attributed to a "truncation artifact."

The $N=3^{(+)}$model that was used to construct the solid line in Fig. 4(c) is obtained by adding a third layer (interface in Table $I$ ) in the immediate vicinity of the silicon-oxide-hydrocarbon interface. The reduced $\chi^{2}$ for this fit, using the 95 data points above $0.1 \AA^{-1}$, is approximately 80 when weighted by Poisson statistics, as compared to a $\chi^{2}$ of approximately 800 for the two-layer fit and 2000 for the one-layer fit for the same points. In view of the facts that (i) the measured reflectivity spans eight or nine orders of magnitude, and (ii) in some cases the statistical weight due to Poisson statistics is less than $0.1 \%$, values of $\chi^{2}$ of the order of 80 could arise from small systematic errors in either the measured signal or the model. In any event, the $N=3^{(+)}$model does account for all of the main features of the data quite well. The main differences between the electron densities of the $N=3^{(+)}$model and the $N=2$ model occur at the silicon-oxide-hydrocarbon interface, with the properties of the other interfaces remaining essentially unchanged. However, the width of the silicon-silicon-oxide interface could still not be determined from the existing data set, the minimum value of $\chi^{2}$ being obtained for an infinitesimally small value for the width of this interface. The fits were carried out with $\sigma_{01}$ arbitrarily set equal to $1 \AA$, the other parameters being relatively independent of the precise value. Similarly, since the data were taken only to $q \leq 0.8 \AA^{-1}$, the results for the fine structure of the silicon-oxide-alkane interface are not unique. The solid line in Fig. $4(\mathrm{c})$ is the best fit for the $N=3^{(+)}$model when $\rho_{2}>\rho_{0}$. The confidence limits listed in Table I for these parameters, as well as the possible variations in the model (i.e., uniqueness) for the silicon-oxide-alkane interface, will be discussed below. Figures 6(a)-6(c) display the electron density as a function of distance from the surface for the $N=1,2$, and $3^{(+)}$models used to calculate the $R(q) / R_{F}(q)$ in Fig. 4. Figure 6(d) illustrates that there are only small quantitative differences between the electron densities for the three models by superposing the three offset curves in Figs. 6(a)-6(c).

In order to assess the confidence limits for the $N=3^{(+)}$ parameters, a set of fits was carried out in which the electron density associated with the interface $\rho_{2}$ was constrained to different values and all other parameters in the model, except for the width of the silicon-siliconoxide interface $\sigma_{01}$, were allowed to vary. Since most of the parameters are tightly coupled, this procedure is necessary to estimate the range of the density $\rho_{2}$ allowed by the data with this model. Figures $7(\mathrm{~b})$ and $7(\mathrm{c})$ display the results for what we subjectively consider to be values of $\rho_{2}$ surrounding the local minimum in $\chi^{2}$ at $\rho_{2} \approx 1.25$ that yield borderline acceptable reflectivity fits. These correspond to values of $\chi^{2}$ that are approximately $25 \%$ larger than the minimum. The $N=3^{(+)}$fit that generated the minimum $\chi^{2}$, Fig. 4(c), is shown again for comparison in Fig. 7(a). The parameters obtained from these fits are listed in Table $I$ as $N=3^{(+1)}$ and $3^{(+2)}$. Similarly, the confidence limits in Table $I$ are arbitrarily set at the values that increase $\chi^{2}$ by approximately $25 \%$, the fits be- ing completed by the same procedure as used to obtain Figs. 7(b) and 7(c).

In order to illustrate the significance of these variations, the real-space electron density for all the three $N=3^{(+)}$models are displayed in Figs. 8(a) $-8(\mathrm{c})$ with all of the interface widths set to be zero, and in Figs. $8(\mathrm{e})-8(\mathrm{~g})$ with the appropriate interface widths. Note that the very high peak density that appears for the second layer in the $3^{(+1)}$ column is misleading since this layer is also very thin. The width of the two interfaces for this layer are similar, resulting in two smeared steps in $d \rho / d z$, of opposite signs, which almost exactly cancel to give the profile shown in Fig. 8(f). Also, the sharp feature in Fig. $8(\mathrm{~g})$ could be smeared out with no appreciable change to the fit quality.

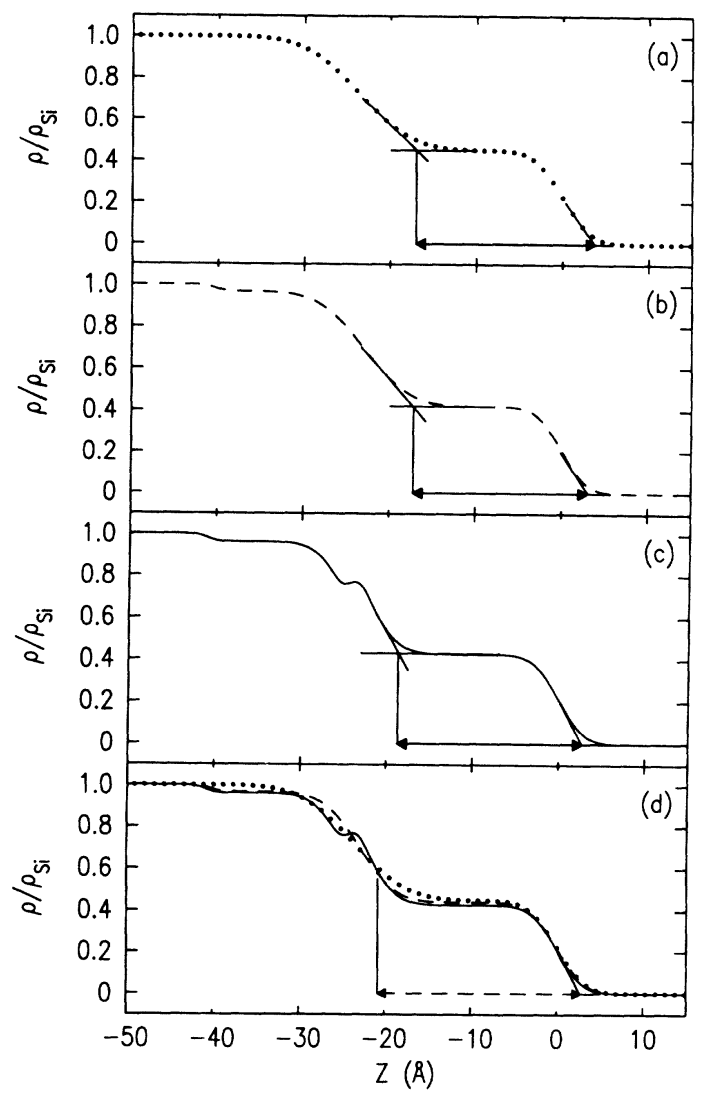

FIG. 6. Real-space profiles of the model surface electron density from the parameters used to obtain fits in Fig. 4. (a) - (c) show the model profiles for the $N=1(\ldots), N=2(---)$, and $N=3$ ( - ) fits. (d) shows the three profiles overlapping for comparison. The hydrocarbon-air interface and hydrocarbon density and length are similar in all the fits, the only region with significant variation being the silicon-oxide-hydrocarbon region. The constructions shown in the three top figures illustrate a graphical technique, which is discussed in the text, for determining the thickness of the hydrocarbon region from these measured densities. The lengths shown as the solid arrows in (a) - (c) are given in Table I. The dashed line in (d) shows the length of $23.6 \AA$ measured directly from the position of the first minimum in the data shown in Fig. 4. 
In addition to the fit shown in Fig. 7(a), there is a second local minimum surrounding the value of $\rho_{2} \approx 0.82$. The best fit parameters for this minimum are listed in Table $I$ in the column $3^{(-)}$and the results for the $R(q) / R_{F}(q)$ are illustrated in Fig. 8(d). In this case, the minimum value of $\chi^{2}$ is about 78 when calculated using the same data as previously.

Although some of the $N=3^{(+)}$and $3^{(-)}$parameters are quite different, the real-space densities, as illustrated in Figs. 8(e) and 8(h), and superposed in Fig. 8(i), have only small quantitative differences only in the region of the $\mathrm{SiO}_{2}$ /alkane interface. In fact, for all of the models described in Table I [i.e., as shown in Figs. 6(d) and 8], the small quantitative differences between the electron densities are much less significant than would appear from the parameters in the table.

That different sets of parameters give rise to similar electron-density profiles suggests that the parameters themselves are not the most meaningful way to interpret the reflectivity data. In the present case, the $N=3$ models were introduced because the reflectivity data clearly indicated that the $\mathrm{SiO}_{2} /$ hydrocarbon interface had some structure. However, since the various model electron densities resultant from these different sets of parameters are similar, the procedure used does allow a relatively unambiguous determination of the electron density responsible for the observed specular reflection. If the interfacial widths were small, the thickness of the hydrocarbon layer could be determined graphically from the distance between sharp breaks in the slope of the curve for $r(z)$. The solid lines in Fig. 6 illustrate one possible way to estimate the corresponding positions when the interfaces have finite width.

We expect that the dominant effect giving rise to the observed interfacial widths is the roughness of the outer $\mathrm{SiO}_{2}$ surface. Assuming that the substrate roughness is

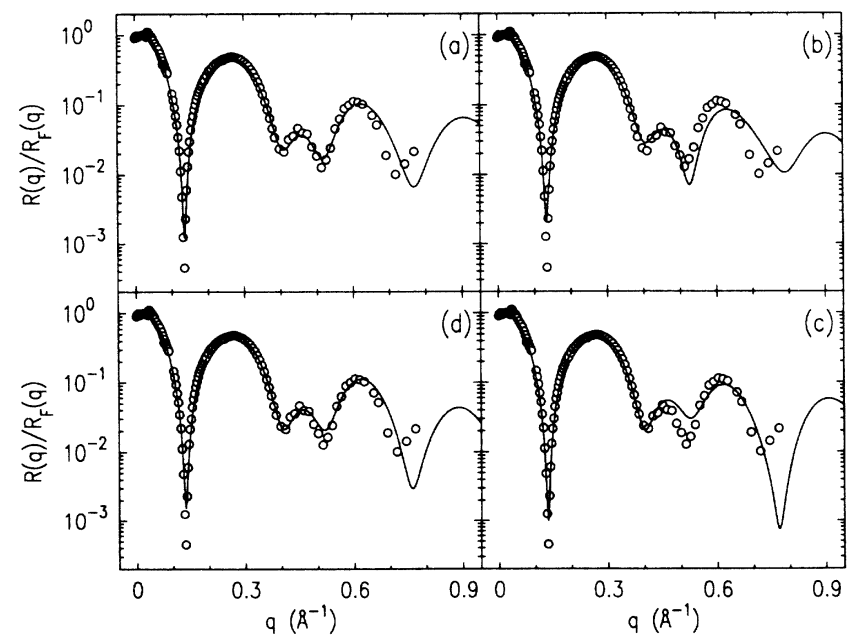

FIG. 7. Comparisons of different fits for the C18 sample whose parameters are shown in Table I. (a) corresponds to column $3^{(+)}$, (b) to column $3^{(-)}$, (c) to column $3^{(+1)}$, and (d) to column $3^{(+2)}$. The parameters are shown in Table $\mathbf{I}$. coated by a fixed thickness of hydrocarbon, the solid construction lines in Fig. 6 illustrate a graphical procedure for determining the average thickness. The results obtained on applying this technique to the models in Figs. 6 and 9 are listed in Table $\mathrm{I}$ in the row $-\left(\mathrm{CH}_{2}\right)-$ graph.
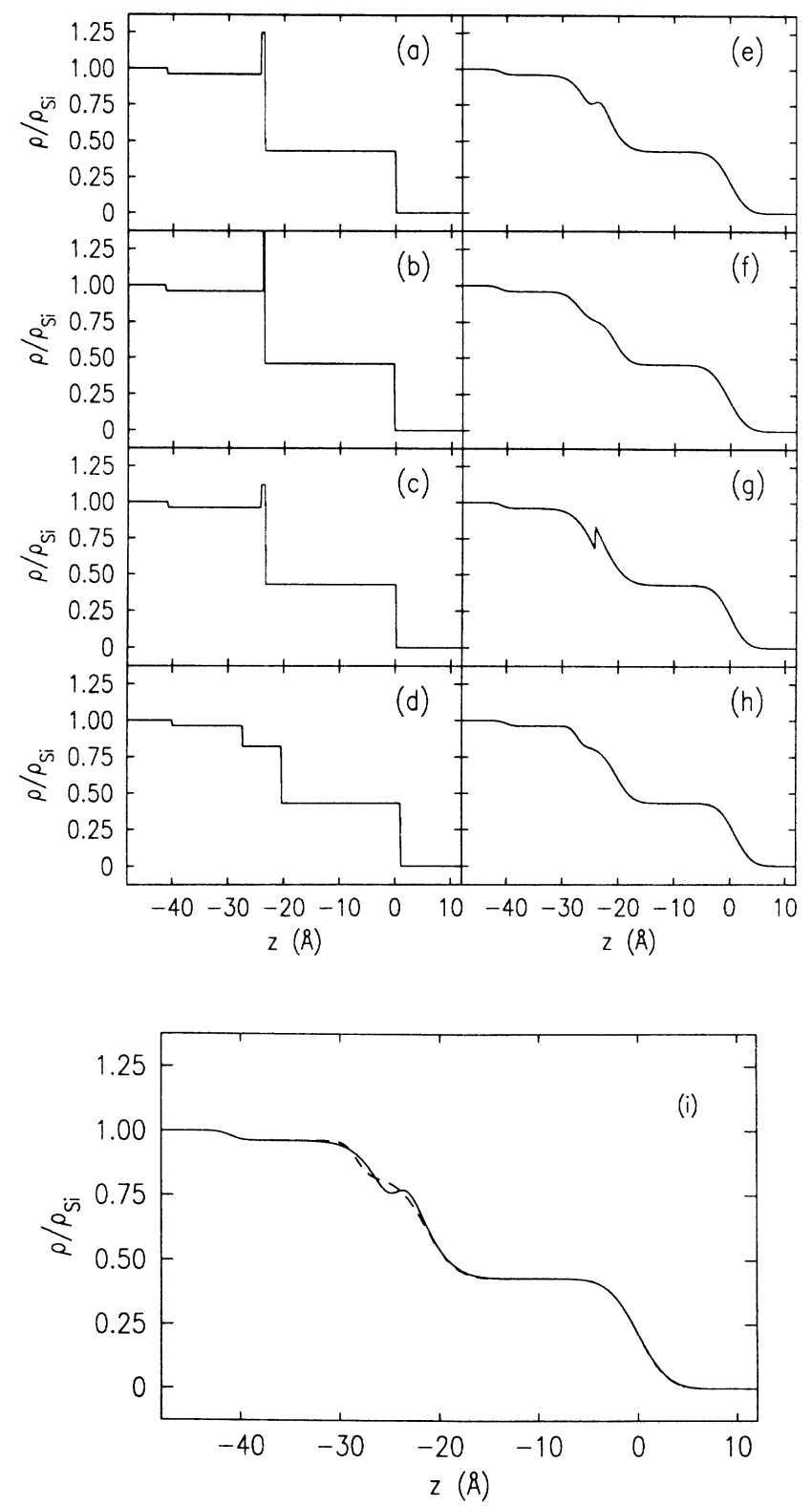

FIG. 8. Real-space densities corresponding to the fits of the reflectivity scans shown in Figs. $7(a)-7(d)$. (a) - (d) show the densities with sharp interfaces. (e) and ( $f$ ) are the corresponding real-space profiles including the widths of the different interfaces. The sharp feature in $(\mathrm{g})$ is well beyond the resolution limit and a fit that is almost as good would have a profile more similar to (e) and (f). (i) shows a comparison of the $3^{(+)}$and $3^{(-)}$ density profiles on the same axes (the two best fits). Although the parameters corresponding to these three curves are quite different, the only significant differences in the real-space densities are in the unresolved structure of the region where the siloxane bonds to the silicon oxide. 

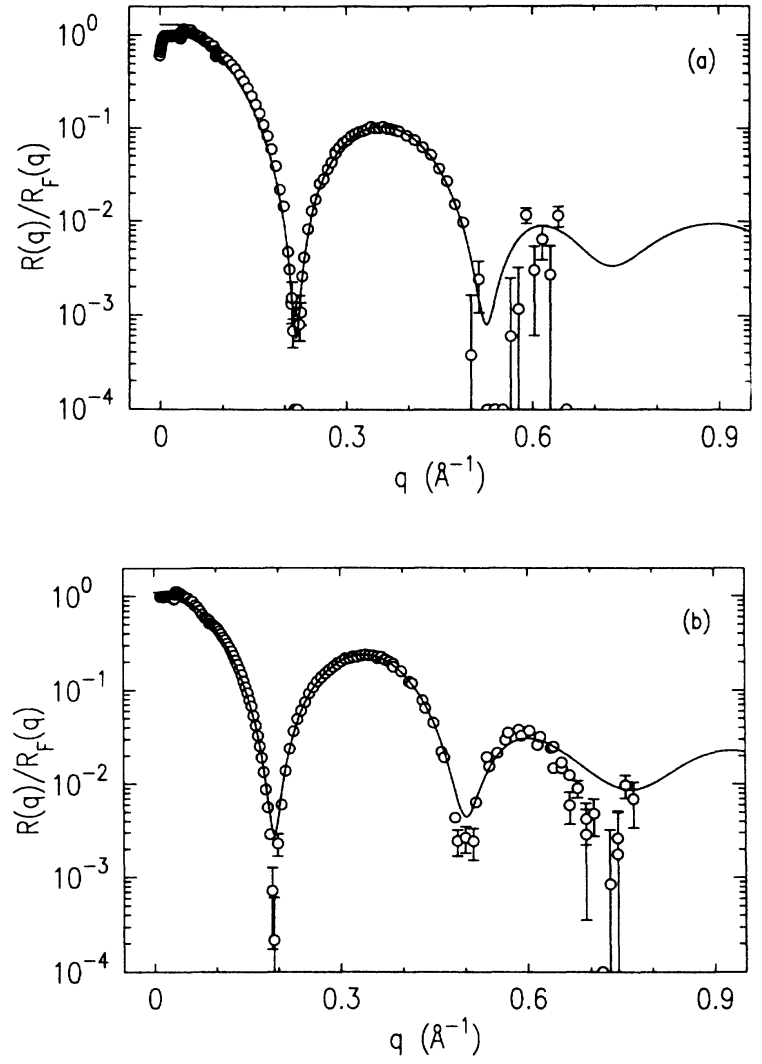

FIG. 9. (a) Three-layer fit and Fresnel normalized reflectivity for a 10-carbon chain alkylsiloxane monolayer. The fit was carried out using 62 data points between $0.18 \AA^{-1} \leq q \leq 0.65 \AA^{-1}$ and, with four adjustable parameters, the $\chi^{2}$ was 9.4. The fit parameters are given in Table II. (b) Three-layer fit and Fresnel normalized reflectivity for a 12-carbon chain alkylsiloxane monolayer. The fit was carried out using 96 data points in the range $0.15 \AA^{-1} \leq q \leq 0.8 \AA^{-1}$ and, with six adjustable parameters, the $\chi^{2}$ was 13 . The fit parameters are given in Table II.

Although there is some variation between the $N=1,2$, and 3 models, the results for the various $N=3$ models are, within errors, identical. While this technique is somewhat arbitrary, we believe it gives a resonable estimate of the length of the hydrocarbon chain excluding the silicon head group. The mean value of $21.3 \pm 0.4 \AA$ is shorter than the length obtained from the position of the dip (23.6 $\AA$ ) (dashed arrow in Fig. 6) due to the specific exclusion of the head group from the graphical length determination.

Since the $N=3^{(+)}$and $3^{(-)}$models give essentially the same structure, we will continue the analysis of the other samples in terms of the model that gives the best fit for that specific sample (holding some parameters fixed if necessary to obtain a physically reasonable structure. This is necessary for those parameters with large uncertainties).

\section{C10 and C12 alkyl chain monolayer}

Further insight into the physical significance of the electron densities obtained from the $N=3$ model can be obtained by consideration of the fits shown in Figs. 9(a) and $9(\mathrm{~b})$, for the $\mathrm{C} 10$ and $\mathrm{C} 12$ coated wafers. Since the reflectivity for the C10 sample was measured only for $q \leq 0.65 \AA^{-1}$, it was not possible to determine either the parameters appropriate to the $\mathrm{SiO}_{2}$ layer or the hydrocarbon electron density from this data set. The fit was thus carried out by assuming that the $\mathrm{SiO}_{2}$ layer for this sample was the same as those of all of the other samples studied. The best parameters for both the $\mathrm{C} 10$ and $\mathrm{C} 12$ fits are listed in Table II and the real-space densities are shown in Fig. 10. The confidence limits for the parameters listed in Table II were set in the same manner as used for the fits for the C18 sample.

The real-space densities for the $N=3^{(+)}$models that provide the "best fit" for the $\mathrm{C} 10, \mathrm{C} 12$, and $\mathrm{C} 18$ coated wafers are displayed superposed on one another in Fig. 10(d). The graphs shown in Fig. 10 suggest that the $\mathrm{SiO}_{2}$ region for the $\mathrm{C} 10$ and $\mathrm{C} 12$ samples might be $\sim 1 \AA$ shorter than that of the C18 wafer. However, since the data for $q \geq 0.5 \AA^{-1}$ are of much lower quality for the $\mathrm{C} 10$ and $\mathrm{C} 12$ than for the C18 sample, we do not believe

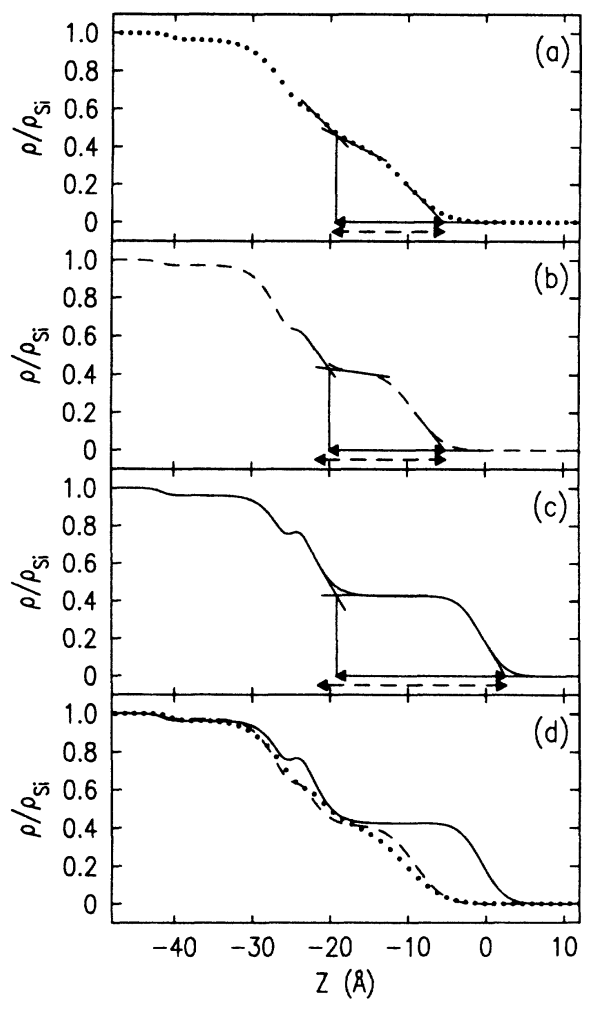

FIG. 10. Real-space profiles for the (a) $\mathrm{C} 10$ (. . . .), (b) $\mathrm{C} 12$ ( -- ), and (c) $\mathrm{Cl} 18$ (_) samples obtained from the reflectivity fits. (d) shows the same profiles overlapping for comparison. Note that although the length of the hydrocarbon layer varies significantly, the silicon oxide layer and layer density are similar for all three samples. The dashed arrows show the length as determined directly from the position of the first minimum. The solid arrows show the construction described in the text used to obtain the revised estimate of the hydrocarbon thickness. 
this difference is necessarily significant. The graphical determinations of the thicknesses of the alkane region for the $\mathrm{C} 10$ and $\mathrm{C} 12$ samples are listed in Table II. from these, we obtain a length per carbon atom of $1.38 \pm 0.2$, $1.23 \pm 0.04$, and $1.18 \pm 0.02 \AA$ for the $\mathrm{C} 10, \mathrm{C} 12$, and $\mathrm{C} 18$ monolayer, respectively. These can be compared with the accepted value for the $1.265 \AA$ for the maximum extension of an aliphatic chain in the all-trans configuration. ${ }^{41}$ Although the $\mathrm{C} 18$ value is slightly shorter, the three results are identical within the quoted errors. This length for the C18 indicates that there could be either a small degree of gauche isomerization or a tilt in the mean axis of the chains with respect to the surface normal. The result allows the layer thickness to be reduced by no more than $10 \%$ from the expected length of an alltrans chain oriented normal to the surface.

As a measure of the packing of the monolayers, it is interesting to calculate the area per alkylsiloxane molecule. Given a length per $\mathrm{CH}_{2}$ group of $1.20 \pm 0.05 \AA$ for the $\mathrm{C} 12$ and $\mathrm{C} 18$ monolayers, a silicon electron density of $7.04 \times 10^{23}$ electrons $/ \mathrm{cm}^{3}$, and a hydrocarbon electron density of $0.42 \pm 0.02$ of that of silicon, one obtains an area-alkylsiloxane molecule of $22.5 \pm 2.5 \AA^{2}$. This area should be compared to an area of $20.5 \AA^{2}$ for long-chain paraffins in bulk ${ }^{44}$ and between 20.5 and $22.5 \AA^{2}$ for Langmuir-Blodgett monolayers of long-chain alcohols. ${ }^{45}$

\section{Partially formed C18 alkyl chain monolayer}

Figure 11 shows the reflectivity from an incomplete $\mathrm{C} 18$ alkylsiloxane film that we designate as C18P. The position of the minima at $q \approx 0.2 \AA^{-1}$ in comparison with $0.13 \AA^{-1}$ for the fully formed C18 film clearly indicates

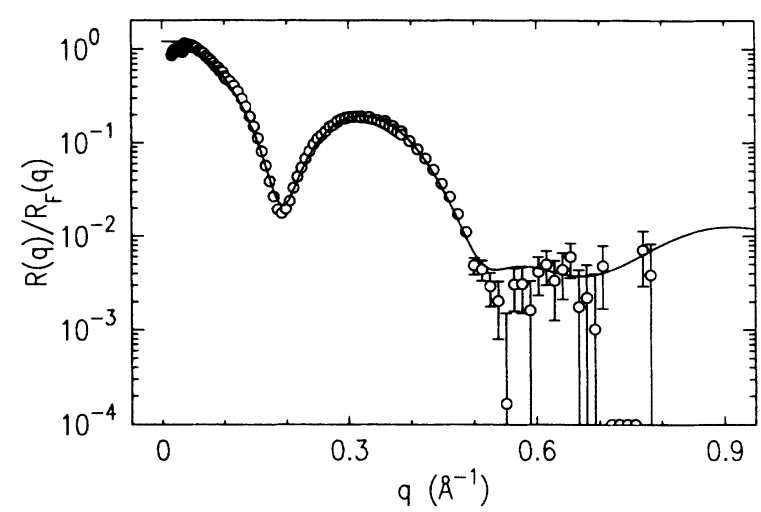

FIG. 11. Three-layer fit and Fresnel normalized reflectivity for a partially formed $\mathrm{C} 18$ alkylsiloxane monolayer. The fit parameters given in Table II were obtained with a $\chi^{2}$ of 32 for points with $q \geq 0.15 \AA^{-1}$.

that this alkylsiloxane film is considerably shorter (i.e., $16.2 \AA$ ) than the fully formed layer $(23.6 \AA)$. The sample reflectivity also falls off much faster than that of the fully formed $\mathrm{C} 18$, suggesting that the alkane-air interface is considerably more diffuse for the partially formed layer.

Detailed analysis of the reflectivity of the C18P monolayer, using the $N=3^{(+)}$model and the silicon oxide layer parameters from previous fits, obtains the fit listed in Table II. The previous observation that the alkane-air interface of the $\mathrm{C} 18 \mathrm{P}$ monolayer is more diffuse than that of the fully formed $\mathrm{C} 18$ is supported by the relative values of $\sigma_{34}$ in Tables I and II. In addition, since the mean value of $\sigma_{34}=3.9 \pm 0.9 \AA$ for the partially formed

TABLE II. Parameters for the $N=3^{(+)}$models that obtain the best representations of the reflectivity for samples as discussed in the text.

\begin{tabular}{|c|c|c|c|c|c|c|c|}
\hline & & $\mathrm{C} 10$ & $\mathrm{C} 12$ & $\mathrm{C} 18 \mathrm{P}$ & $\mathrm{C} 17^{(=)}$ & $\mathrm{C} 17^{(\mathrm{Br})^{\mathrm{a}}}$ & $\mathrm{CF}$ \\
\hline \multicolumn{8}{|c|}{ Layer thickness $L_{j}(\AA)$} \\
\hline $\mathrm{SiO}_{2}$ & $L_{1}$ & $16.0 \pm 1.5$ & $16.6 \pm 0.8$ & $17.0^{\mathrm{b}}$ & $12.7 \pm 2.5$ & $12.7^{\mathrm{b}}$ & $16^{\mathrm{b}}$ \\
\hline Interface & $L_{2}$ & $1.3 \pm 1.5$ & $0.6 \pm 0.6$ & $0.4_{-0.4}^{+3.0}$ & $6.1 \pm 1.5$ & $6.1^{\mathrm{b}}$ & 2.0 \\
\hline$-\left(\mathrm{CH}_{2}\right)-$ & $L_{3}$ & $13.0 \pm 0.3$ & $15.6 \pm 0.2$ & $16.5 \pm 1.0$ & $20.1 \pm 1.5$ & $23.7 \pm 0.2$ & 0.1 \\
\hline$-\left(\mathrm{CF}_{2}\right)-$ & $L_{4}$ & & & & & & 12.0 \\
\hline$-\left(\mathrm{CH}_{2}\right)-\mathrm{graph}$ & $L_{3}$ & $13.8 \pm 2.0$ & $14.8 \pm 0.5$ & $17.2 \pm 0.4$ & $19.9 \pm 0.4$ & & $18 \pm 2^{c}$ \\
\hline \multicolumn{8}{|c|}{$\rho_{j} / \rho_{0}$} \\
\hline $\mathrm{SiO}_{2}$ & $\rho_{1}$ & $0.97^{b}$ & $0.97 \pm 0.01$ & $0.98 \pm 0.01$ & $0.98 \pm 0.01$ & $0.98^{b}$ & $0.98^{\mathrm{b}}$ \\
\hline Interface & $\rho_{2}$ & $0.30_{-0.08}^{+15}$ & $0.18_{-0.15}^{+10}$ & $1.13_{-0.15}^{+5.0}$ & $0.78 \pm 0.15$ & $0.78^{\mathrm{b}}$ & $0.43^{b}$ \\
\hline$-\left(\mathrm{CH}_{2}\right)-$ & $\rho_{3}$ & $0.41_{-0.04}^{b+0.2}$ & $0.41_{-0.02}^{+0.04}$ & $0.40 \pm 0.08$ & $0.41 \pm 0.02$ & $0.41^{b}$ & 0.77 \\
\hline$-\left(\mathrm{CF}_{2}\right)-$ & $\rho_{4}$ & & & & & & 0.80 \\
\hline \multicolumn{8}{|c|}{$\sigma_{i j}(\AA)$} \\
\hline $\mathrm{Si} / \mathrm{SiO}_{2}$ & $\sigma_{01}$ & $1^{\mathrm{b}}$ & $1^{\mathrm{b}}$ & $1^{\mathrm{b}}$ & $1^{\mathrm{b}}$ & $1^{\mathrm{b}}$ & $1^{\mathrm{b}}$ \\
\hline $\mathrm{SiO}_{2} /$ Inter. & $\sigma_{12}$ & $4.2_{-0.2}^{+1.0}$ & $3.3 \pm 0.5$ & $1^{\mathrm{b}}$ & $2.2 \pm 1.2$ & $2.2^{\mathrm{b}}$ & 4.2 \\
\hline Inter $/\left(\mathrm{CH}_{2}\right)$ & $\sigma_{23}$ & $1^{\mathrm{b}}$ & $1^{b}$ & $3.4 \pm 1.5$ & $3.4 \pm 1.2$ & $3.4^{\mathrm{b}}$ & 2.8 \\
\hline$\left(\mathrm{CH}_{2}\right) / \mathrm{Air}^{\mathrm{d}}$ & $\sigma_{34}$ & $3.8 \pm 0.3$ & $2.9 \pm 0.3$ & $3.9 \pm 0.9$ & $3.0 \pm 0.2$ & $2.1 \pm 0.5$ & $1^{\mathrm{b}}$ \\
\hline$\left(\mathrm{CF}_{2}\right) / \mathrm{Air}$ & $\sigma_{45}$ & & & & & & 2.9 \\
\hline
\end{tabular}

${ }^{\mathrm{a}} L_{\mathrm{Br}}=-4.8 \pm 0.6 \AA, \sigma_{\mathrm{Br}}=2.4 \pm 0.4 \AA, n_{\mathrm{Br}}=1.40 \pm 0.15\left(\AA \rho / \rho_{0}\right)$.

${ }^{b}$ Parameters kept fixed during fitting.

${ }^{c}$ Combined length of the fluorocarbon and hydrocarbon chains.

${ }^{\mathrm{d}}$ For the fluorocarbon sample $\mathrm{CF}$ this is the $\left(\mathrm{CH}_{2}\right) /\left(\mathrm{CF}_{2}\right)$ interface. 
$\mathrm{C} 18 \mathrm{P}$ monolayer is noticeably larger than $\sigma_{34}=2.9 \pm 0.3$ $\AA$ for the fully formed $\mathrm{C} 12$ layer with comparable thickness to the C18P sample, there is a high probability that the alkane-air interface is considerably more diffuse for the partially formed layer than for either of the two potentially similar systems. The fit for the $\mathrm{C} 18 \mathrm{P}$ monolayer is illustrated by the solid line in Fig. 11 .

That the electron density of the partially formed layer is comparable to the density obtained for the fully formed layer suggests that the alkane chains either tilt, or otherwide bend, to fill space in order to maintain a density close to the fully formed hydrocarbon density of approximately $0.85 \mathrm{gm} / \mathrm{cm}^{3}$. If the partial monolayer was comprised of close packed, uniformly tilted, straight C18 chains, the mean tilt would be about $45^{\circ}$ if one uses the lengths obtained from the dip position or $36^{\circ}$ using the graphically determined length. These results are not consistent with one previously suggested model of partially formed films as islands of close-packed, straight, fully extended molecules that are oriented normal to the surface. ${ }^{46,47}$ The average electron density for this model would consist of a layer of the same length but a lower electron density. Variations of this model, in which the molecules at the boundary of the islands were partially disoriented, would increase the apparent interfacial width $\sigma_{34}$, but would not change the thickness.

Figure 12 shows the comparison of the alkylsiloxane lengths as measured by ellipsometry and from $2 \pi / q_{\min }$, where $q_{\min }$ is the position of the first minimum in the $\mathrm{x}$ ray reflectivity. The ellipsometric measurements will be described in more detail in a separate publication. ${ }^{34}$ The $\mathrm{x}$-ray measurements were made using both the rotating anode and synchrotron sources. Assuming a constant

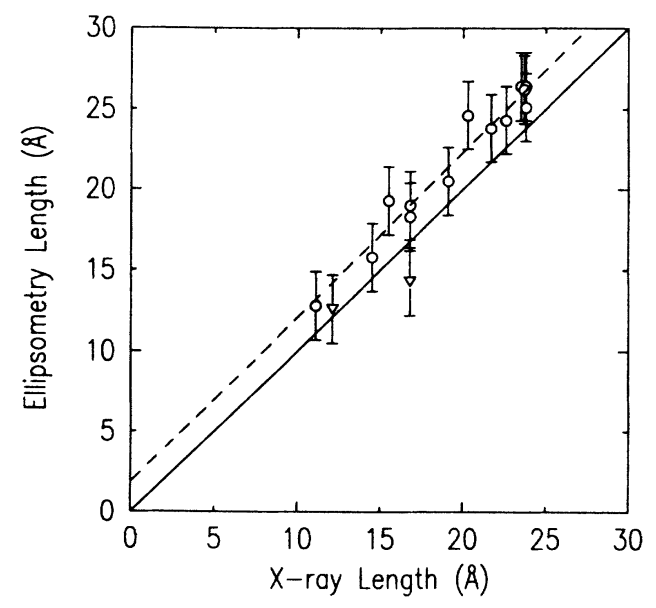

FIG. 12. Comparison of the alkylsiloxane length as determined by $x$-ray reflectivity and by ellipsometry. ( $O$ ) are fully formed monolayers, $(\nabla)$ are incompletely formed layers. The solid line corresponds to the expected curve if both techniques gave the same result. The dashed curve is a fit to the fully formed layer results and has the form $L^{\text {ellip }}=1.02( \pm 0.06)$ $\times L^{\mathrm{x} \text { ray }}+1.8( \pm 1.0) \AA$. The two techniques appear to predict the same length per $\mathrm{CH}_{2}$ group, but have different sensitivities to the silicon oxide-hydrocarbon interface. offset, the average difference between the ellipsometrically determined length and $2 \pi / q_{\min }$ corresponds to $1.8 \pm 1.0 \AA$ with the ellipsometric value being larger. Since the graphically determined value for the $x$-ray determined thickness is of the order of $1.5 \pm 0.6 \AA$ shorter than $2 \pi / q_{\min }$, the ellipsometric values are of the order of $3.0 \AA$ larger than the graphically determined values. This difference is slightly outside of the quoted errors of approximately $\pm 0.5 \AA$ in the $x$-ray and $\pm 2 \AA$ for the ellipsometrically measured lengths and may be systematic, having an origin in factors such as the effects of the interface on either technique, size related corrections to the index of refraction for the ellipsometric technique, etc.

\section{Other samples}

\section{Bromination of alkene terminated sample}

This sample was prepared initially as a $\pi$-bond terminated siloxane sample with 17 carbon atoms [i.e., $\left.\mathrm{Si}-\left(\mathrm{CH}_{2}\right)_{15}-\mathrm{CH}=\mathrm{CH}_{2}\right]$. The data, in the form of $R(q) / R_{F}(q)$, and the calculated results for the $N=3^{(+)}$ model are shown in Fig. 13(a). The parameters for the fit are displayed in Table II in the column $\mathrm{C}_{17} 7^{(=)}$and the real-space electron density is illustrated by the broken line in Fig. 14. The reflectivity data are substantially the same as for the simple alkane samples except for a somewhat more diffuse hydrocarbon-air interface $(3.0 \AA$ versus approximately $2.5 \AA$ for the simple C 18 molecule of similar length), but aside from this variation, there are no systematic differences between the real-space electron densities extracted from this data set and the one for the

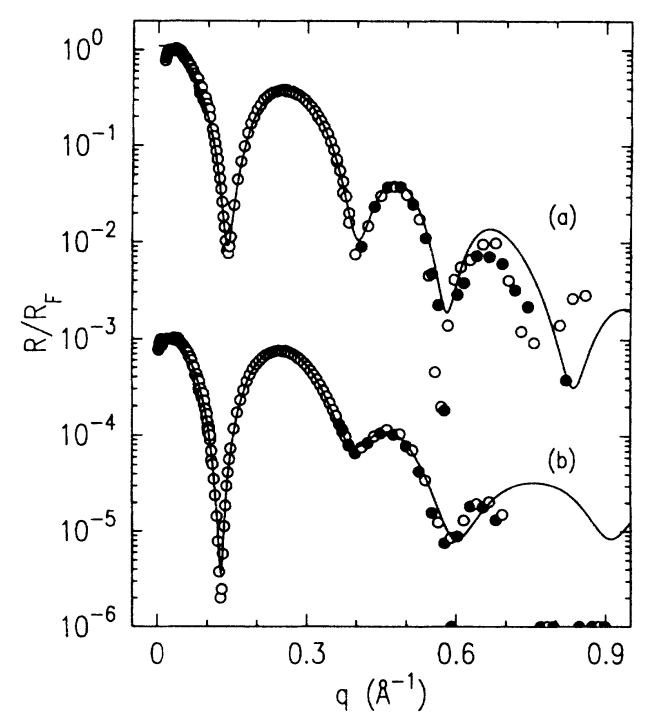

FIG. 13. (a) Fresnel normalized reflectivity from the olefin terminated sample together with (b) the reflectivity from the same sample after bromination. The technique used to fit the data is described in the text, with the parameters for the fits given in Table II. The fits for (a) and (b) have $\chi^{2}$ values of 33 and 60 for the points above $0.1 \AA^{-1}$. The filled points at large $q$ indicate the data taken on a second measurement. The small systematic differences may be indicative of the radiation damage also observed by the contact angle and XPS measurements. 


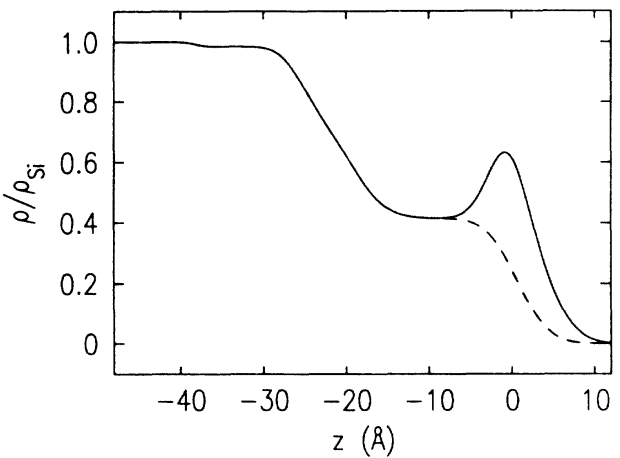

FIG. 14. Real-space electron density profile of the surface obtained from the parameters of the fits in Fig. 13 for the alkene sample and the same sample after bromination. Note that the fit to the brominated sample was done by using the parameters of the unbrominated sample (dashed line) for all except the hydrocarbon-air interface. The addition of the peak is due to the addition of one bromine atom to each of the two carbon atoms at the tail of the molecule.

\section{C18 sample.}

After the initial $x$-ray measurement, the same sample was brominated and measured again [Fig. 13(b)]. Bromination results, to a first approximation, in breaking the terminal $\pi$ bond and attaching two bromine atoms to the two terminal carbon atoms to give the structure $-\mathrm{Si}-\left(\mathrm{CH}_{2}\right)_{15}-\mathrm{CHBr}-\mathrm{CH}_{2} \mathrm{Br}$. Relative to the data in Fig. 13(a), the overall reflectivity has increased, suggesting the presence of additional electron density at the surface, and the position of the first minimum has shifted to a lower angle as would occur if the distance between surfaces were increased.

More detailed fitting was carried out by considering the addition of a single Gaussian to the real-space electron density profile to the $N=3^{(+)}$model that described the $\mathrm{C} 17^{(=)}$data, to account for the bromine electron density. The three new adjustable parameters in fitting the $\mathrm{C} 17^{(=)}$data were the position

$$
D_{\mathrm{Br}} \equiv \sum_{i=1}^{3} L_{i}+L_{\mathrm{Br}},
$$

the width $\sigma_{\mathrm{Br}}$, and the area $n_{\mathrm{Br}}$ of the Gaussian. The fit was carried out by holding fixed most of the other parameters at the values in column $\mathrm{C} 17^{(-)}$of Table II and allowing only $L_{3}, L_{\mathrm{Br}}, \sigma_{34}, \sigma_{\mathrm{Br}}$, and $n_{\mathrm{Br}}$ to vary. The values for the parameters that gave the best fit are listed in column $\mathrm{C} 17^{(\mathrm{Br})}$ of Table II and in the notes below the table. The solid line in Fig. 14 displays the real-space electron density for the $\mathrm{C} 17^{(\mathrm{Br})}$ sample that gave rise to the model reflectivity illustrated by the solid line in Fig. 13(b). The combination of a larger value of $L_{3}$ for the $\mathrm{C} 17^{(\mathrm{Br})}$ and the Gaussian (centered slightly below the surface as indicated by a negative $L_{\mathrm{Br}}$ ) is shown in Fig. 14 to place the additional electrons of the $\mathrm{Br}$ atoms close to the end of the alkane chains. The net effect is to extend the overall thickness of the monolayer.

From the extra area associated with the addition of the bromine atoms we calculate the addition of $45 \pm 10$ electrons per alkylsiloxane group. Assuming that bromination takes place as described above, the fully brominated layer would have 66 effective electrons per alkylsiloxane (the two $1 s$ electrons per bromine atom are too tightly bound to contribute to the measured electron density). This measurement thus implies that only $\frac{45}{66}$, or $68 \%$ of the molecules were brominated.

XPS analysis carried out some weeks later than the $\mathrm{x}$ ray measurement showed that on sections of the sample which were not in the beam $90 \%$ of the molecules were brominated, but on radiated sections this figure was only about $30 \%$. It is clear that the radiation had initiated some chemical change to the monolayer surface. Damage occurred during the $\mathrm{x}$-ray exposure both before and after bromination. This damage is visible in the reflectivity scans shown in Fig. 13 as a systematic shift of data taken 30 min later at large scattering vectors (shown as solid symbols). While this implication of $\mathrm{x}$-ray damage adds uncertainty to the significance of the $x$-ray determined structure, it does not alter the basic objective of demonstrating that specular reflection can be used for quantitative determination of chemical modifications of the alkane surface.

\section{Fluorocarbon coated sample}

In order to demonstrate the applicability of the technique to samples with a radically different layer density from the hydrocarbon, a wafer was coated with a monolayer of $-\mathrm{Si}-\left(\mathrm{CH}_{2}\right)_{2}-\left(\mathrm{CF}_{2}\right)_{7}-\mathrm{CF}_{3}$. The reflectivity data for this sample, in the form of $R(q) / R_{F}(q)$, and the calculated results for an $N=4$ model are shown in Fig. 15(a). Since the difference in electron density between the silicon oxide and the fluorocarbon layers is much less than that between the silicon oxide and the alkane layer in the previous samples, the amplitude of the $x$ rays reflected from the fluorocarbon-air interface is correspondingly stronger than the net amplitude reflected from the composite interface between the fluorocarbon-silicon-oxide interface. As a consequence, the depth of the first interference minimum at $q \approx 0.2$ $\AA^{-1}$ is much shallower in this sample than the corresponding minimum for the alkane coated samples. On the other hand, since the amplitude of the signal reflected from the fluorocarbon-air interface is greater than the amplitude of the signal from the composite interface, and since that is yet larger than the amplitude reflected from the $\mathrm{Si} / \mathrm{SiO}_{2}$ interface, the interference pattern is dominated by the two signals from the first two interfaces. As a result, the two minima at $q=0.18$ and $0.55 \AA^{-1}$ in Fig. 15 (a) correspond to roughly $q L=\pi$ and $3 \pi$ with $L \approx 17.8$ $\AA$. We suggest that this is the distance between the silicon-oxide $/\left(\mathrm{CH}_{2}\right)_{2}$ and the fluorocarbon-air interface. This length should be compared to a length of the $18.1 \AA$ obtained from the graphical analysis of the four-layer fit.

The one unfortunate consequence to follow from the fluorocarbon electron density is that the reflectivity is less sensitive to the $\mathrm{SiO}_{2}$ layer and its two interfaces. The solid line in Fig. 15(a) is calculated from a model in which $N=4$, but using values for the parameters describ- 

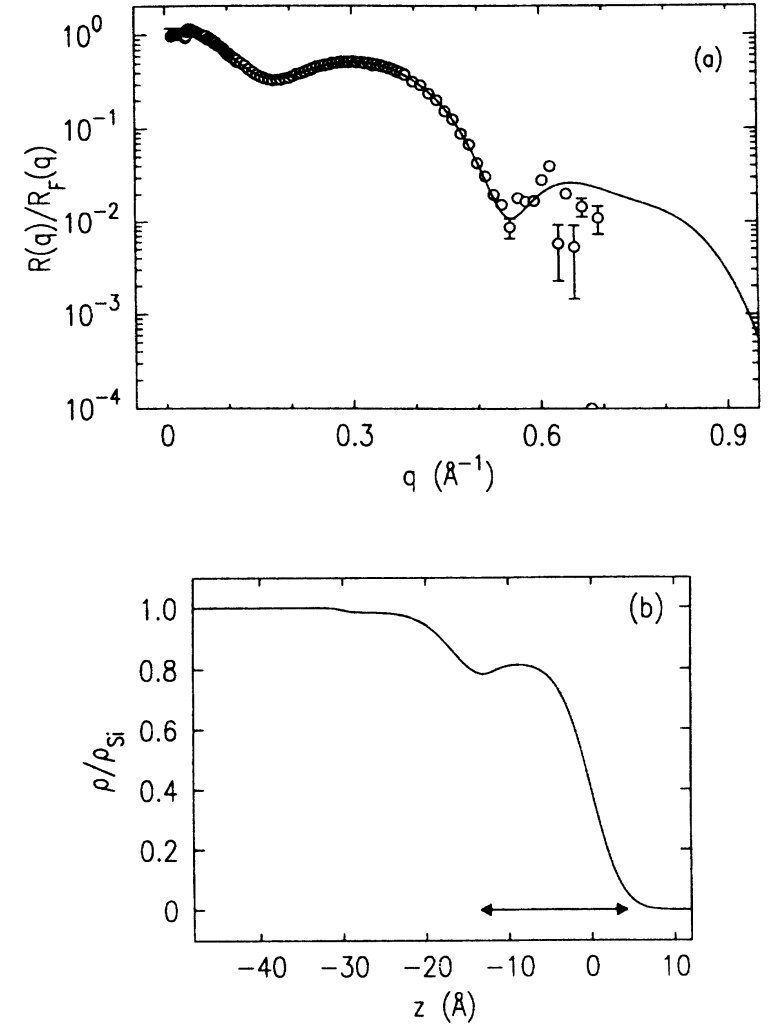

FIG. 15. (a) Normalized reflectivity and three-layer fit for a fluorocarbon sample. The parameters are shown in Table II. Because of the limited range of data and the complicated nature of the interface, it was impossible to obtain accurate values for the parameters, the fit being only a physically reasonable one. (b) Real-space electron density profile of the surface of the sample obtained from the parameters of (a). Note that the fluorocarbon chain has a much higher density than the hydrocarbon chain resulting in the less pronounced first minimum. The dip in the real-space profile corresponds to the location of the two methylene $\left(\mathrm{CH}_{2}\right)$ groups in the molecule.

ing the $\mathrm{SiO}_{2}$ layer, its interfaces and other parameters for the hydrocarbon portion of the molecule as determined from the other samples. In addition, since the data were taken only for $q \leq 0.7 \AA^{-1}$ and since the points near $q \approx 0.7 \AA^{-1}$ themselves have large error bars, the confidence limits on the fit parameters are larger than those for the other fits. In any event, the parameters that obtained the best fit and are physically realistic are displayed in Table II. The real-space electron density is shown in Fig. 15(b). The solid line indicates the $17.8 \AA$ that is the origin of the principle interference minima in Fig. 15(a).

\section{DISCUSSION}

The very deep nature of the first interference minimum for the hydrocarbon samples with well formed films is a direct demonstration that organic monolayers synthesized by the self-assembly process are capable of pro- viding microscopically and macroscopically uniform films. In particular, the first sharp minimum in the reflectivity allows an approximate determination of the thickness of the adsorbed film that is in reasonable agreement with the thicknesses predicted by assuming maximally extended aliphatic chains normal to the surface, when the size of the silicon head group is assumed to be included in the length measured. Comparisons between $\mathrm{x}$-ray reflectivities calculated from more detailed models, which included structure in the head region, gave a hydrocarbon thickness that also suggests maximally extended molecules. The principal residual uncertainty of the hydrocarbon thickness is due to the width of the interfacial region between the $\mathrm{SiO}_{2}$ layer and the hydrocarbon layer. We suspect that the major contribution to this width is the roughness of the bare $\mathrm{SiO}_{2}$ substrate and that a significant improvement could be obtained by preselection of flatter substrates.

The highly sensitive dependence of the reflectivity on details of the monolayer structure is indicated by the much improved fits of the $N=3$ model as compared to the $N=2$ model, the differences being only small changes in the electron-density profile at the silicon oxide-alkylsiloxane interface. The reflectivity is particularly sensitive to the interface width, which must be considered separately for each of the interfaces if a good fit to the data is to be obtained. This sensitivity to interfacial structure has been neglected in most other $x$-ray studies of similar systems.

Inspection of the various models for the 18 carbon alkylsiloxane real-space electron density (i.e., Fig. 6) suggests the hypothesis that the real-space density might be the result of coating the $\mathrm{SiO}_{2}$ surface, which has some roughness, with a fixed thickness of alkane. The variations in the model parameters for the width of the $\mathrm{SiO}_{2}$ /alkane and alkane-air interfaces leads to some uncertainty in the thickness to the alkane layer. However, from the graphical inspection of the various models described earlier, we believe that this uncertainty is no more than $\pm 0.5 \AA$ (1.0 $\AA$ for the C10 alkylsiloxane). Using the thickness of the hydrocarbon part of the C18 molecule only, the measured tilt angle is about $\cos ^{-1}[21.4 /(1.265 \times 18)]=20^{\circ} \pm 4^{\circ}$.

In summary, the $\mathrm{x}$-ray data are consistent with uniform monolayers whose thicknesses are of the order of 95\% of the expected values for maximally extended alkane chains normal to the surface. The layer thicknesses determined directly from the dip minimum in the $x$-ray specular reflectivity also agree within $2 \AA$ with those determined by ellipsometric measurement. Since the width of the head group affects the position of the minimum, we believe, however, that the thickness of the alkane region alone may be as much as $4 \AA$ thinner than the ellipsometrically determined values.

The structure near the silicon-oxide-hydrocarbon interface is almost certainly due to the silicon-oxygen network formed by the siloxane head groups to neighboring atoms and to the silicon oxide surface. Since the available data are restricted to a region of $q \leq 0.8 \AA^{-1}$, we did not have sufficient resolution to distinguish between a well-formed head-group layer, at a fixed distance from 
the $\mathrm{SiO}_{2}$ substrate, or one that is more distorted. From the absence of any chlorine signal in the XPS spectra, we can, however, be confident that the interface structure is not due to chlorine atoms remaining from the preparation. ${ }^{40}$

The electron density of the fully formed alkylsiloxane layers was essentially independent of the sample, having values between 0.41 and 0.43 of the silicon electron density. This value is equivalent to a mass density of between 0.83 and $0.87 \mathrm{~g} / \mathrm{cm}^{3}$ (silicon has a mass density of 2.33 $\left.\mathrm{g} / \mathrm{cm}^{3}\right)$. This is somewhat larger than densities of liquid phases of alkanes containing $12-18$ carbon atoms $(0.75$ and $0.78 \mathrm{~g} / \mathrm{cm}^{3}$ ), but it is less than the densities found for crystalline phases of the same materials $\left(0.93 \mathrm{~g} / \mathrm{cm}^{3}\right) .{ }^{44}$ This is equivalent to the statement that the area per hydrocarbon chain was found to be approximately $10 \%$ larger than that of crystalline alkanes.

The width of the hydrocarbon-air interface does vary considerably between the long and short hydrocarbon chains, with values of $3.8,2.9$, and $2.4 \AA$ for the $\mathrm{C} 10$, C12, and $\mathrm{C} 18$ alkylsiloxanes, respectively (see Fig. 10). This variation may be due to the increased flexibility of the longer chains which are able to deform more easily and thereby quench some of the nonuniformity introduced at the silicon-oxide-hydrocarbon interface.

The most striking feature of the partially formed $\mathrm{C} 18$ alkylsiloxane reflectivity data when compared to the fully formed layer is the change in the position of the first minimum, unambiguously indicating a reduced thickness of the incomplete monolayer. Since the average electron density in the alkane layer is essentially the same as that for all of the fully formed layers, this result rules out the previously suggested model of islands of fully extended molecules. ${ }^{32}$ Since, for films thinner than approximately $100 \AA$, ellipsometry is only sensitive to the mean optical thickness, this technique cannot distinguish between the island hypothesis and the uniform layer. The reduced thickness of the partially formed layer could be explained in terms of a homogeneous coating in which the mean molecular tilt was about $45^{\circ}$. However, since the alkane-air interface for the incompletely formed monolayer was also found to be rougher than the same interface for all of the fully formed monolayers, the correct description of the partially formed monolayer must involve some degree of nonuniformity in the coating.

The present set of measurements cannot distinguish between a uniform-diffuse interface and one that was microscopically sharp, but rough. In principle, this distinction could be made by a more systematic study of the line shape as $\phi$ is tuned off of the specular condition, like those shown in Fig. 3, but using a much finer resolution such as can be obtained using a crystal analyzer. ${ }^{14}$ The data reported here will not support a value for the parameter $\sigma_{01}$ that describes the $\mathrm{Si} / \mathrm{SiO}_{2}$ interface that is greater than $2 \AA$. The fitting algorithm always drives it to zero.

A variation of the technique described here is to study the way the intensity falls off as the spectrometer is tuned away from the condition for Bragg reflection rather than away from the condition for specular reflection. The intensity along the "truncation rods" ${ }^{48,49}$ can be interpret- ed in terms of the width of the termination of the crystal lattice rather than as we have done in terms of the density profile at the interface between two regions of differing average electron density. The data for the surface of crystalline silicon indicate that the silicon lattice termination occurs in a single step, giving an atomically flat silicon-silicon-oxide interface. ${ }^{48,49}$ Another study using transmission electron diffraction on specially prepared silicon wafers also found perfect termination of the silicon crystal lattice. ${ }^{50}$ A 5 - $\AA$ layer of ordered silicon oxide crystal was found at the crystalline silicon-amorphous silicon-oxide interface. Our measurements are consistent with these results indicating a very narrow silicon-silicon-oxide interface. This interface and, in particular, its width are of exceptional importance to the silicon-based electronics industry. 42,51

Using other more intense synchrotron beam lines, it should be straightforward to extend $x$-ray reflectivity measurements to values of $q$ at least two or three times larger than the maximum of $0.8 \AA^{-1}$ in the present measurements. Provided that neither radiation damage nor diffuse background radiation are the limiting features, the structure of the various interfaces could be determined to a spatial resolution that could be three times finer than achieved in the present measurement. Furthermore, if the $\mathrm{Si} / \mathrm{SiO}_{2}$ sample is protected from airborne contaminants, specular reflectivity measurement of the bare $\mathrm{Si} / \mathrm{SiO}_{2}$ substrate is the only method of which we are aware that has the potential for fully characterizing the transition from the crystalline silicon region, through the strained crystalline $\mathrm{Si} / \mathrm{SiO}_{2}$ region, into the region of amorphous $\mathrm{SiO}_{2}$.

In some respects the use of the $\mathrm{Si} / \mathrm{SiO}_{2}$ substrate, with its native oxide layer, complicated the analysis and made it more difficult to characterize the alkane surfaces. On the other hand, the $\mathrm{Si} / \mathrm{SiO}_{2}$ substrates have the decided advantages of having a much sharper interface with air than any other solid surface of comparable dimensions that we could obtain. In addition, since the observed diffuse scattering from the $\mathrm{Si} / \mathrm{SiO}_{2}$ samples, at all angles of incidence, is significantly lower than that observed from highly polished amorphous materials (such as polished or float glass), we believe that the microscopic surface width of the $\mathrm{Si} / \mathrm{SiO}_{2}$ surface is also significantly less than that of other possible substrates.

The data on the olefin-terminated $\mathrm{C} 17$ sample, together with the study on the effect of bromination, illustrate a powerful tool for study of certain types of surface structures. In this particular example $\mathrm{Br}_{2}$ molecules added to the reactive olefin groups attached to the end of the alkyl chains. From the $x$-ray reflectivity, it was possible to observe the position of the additional electrons and the consequent distribution of the bromine atoms. Although there were some effects of radiation damage that were discovered after the x-ray measurements had been completed, the example suggests that this type of measurement could be carried out using a much larger variety of reactive species. In particular, if the moiety to be attached to the end of the alkane group has some extended structure, this technique would allow for a relatively detailed mapping of its electron density. Since the specular 
reflection from the substrate provides a reference field, this technique has a built-in solution to the phase problem that plagues most other $\mathrm{x}$-ray techniques for structural determination.

The most serious limitation on the potential applicability of specular reflection for the study of organic monolayers is the problem of radiation damage. In the present work most of the samples with well-formed alkanes had contact angles with water of approximately $110^{\circ}$ before synchrotron reflectivity measurement and from $75^{\circ}$ to $90^{\circ}$ afterwards depending on the amount of exposure. XPS studies of the irradiated region suggested between $5-20 \%$ of the alkyl chains had been oxidized, while sections of the sample that were not irradiated did not show the presence of any oxidized species. ${ }^{34}$ This damage was not observed on samples measured during experiments using the rotating anode with filtered radiation (where total x-ray exposure was roughly $1 \%$ of the synchrotron exposure), but was reproduced when a sample was exposed to the polychromatic beam for $24 \mathrm{~h}$. In a few cases, there was some evidence that for $q \geq 0.7 \AA^{-1}$ radiation damage may have been observed as changes in the reflectivity of the order of $30 \%$. However, no changes were detected for $q \leq 0.5 \AA^{-1}$.

Since only about 0.1 photons per alkyl group fell on the sample during the course of a typical series of scans, with most being transmitted into the silicon bulk, and given that the number of damaged molecules exceeds this number, the damage cannot be associated with the photoelectric effect acting directly on the alkylsiloxane molecules. One possibility is that the damage is induced by photoelectrons generated by $\mathbf{x}$ rays in the silicon substrate. These keV energy electrons interact strongly with other electrons spawning many more secondary electrons which could ionize the carbon atoms in the alkylsiloxane chain. The final damage would then result when the highly reactive radicals thus formed combine with oxygen in the surrounding air. Alternatively, $x$-ray induced ozone in the atmosphere might be the source of the damage, or there might be free-radical chain reactions mediated by oxygen in the organic monolayers.

There are a number of ways that this damage might be reduced. The first and most obvious way would be to place the sample in an atmosphere free of $\mathrm{O}_{2}$. The second would be to make the measurements with significantly less exposure to radiation than was the case in these studies. For example, measurements of background scattering do not necessarily have to be taken with the same statistics as for the main data. In any event, the background scans can be taken after the reflectivity data have been completed. Since the background is mainly due to air and bulk scattering, it will be little affected by surface damage. The exposure can also be reduced by optimizing the number of data points to eliminate much of the redundancy evident in the data in Fig. 1 at low angles. In addition, since the samples have proven to be highly uniform, data points can be taken on the different regions of the wafer.

Finally, the changes in the reflectivity with time that were measured on the uncoated silicon sample are probably due to the build up on the surface of a contamination layer with a density lower than silicon. The strong signal from this layer makes it difficult to characterize the $\mathrm{SiO}_{2}$ layer from the data in Fig. 1(a), and future measurements of the uncoated $\mathrm{Si} / \mathrm{SiO}_{2}$ surface must be done under more rigorously controlled conditions than were available for this study.

\section{ACKNOWLEDGMENTS}

The authors express their thanks to D. Osterman for help during the work at NSLS. This work was supported by the National Science Foundation through Grants to the Harvard Materials Research Laboratory, No. NSFDMR-85-13523 and No. NSF-DMR-86-14003, from the Joint Services Electronics Program of the Department of Defense through Grant No. N0014-84-K-0465, and from the U.S. Office of Naval Research and U.S. Defense Advanced Research Projects Agency Grants No. N0001483-K-0142 and No. N00014-85-K-0898. Research carried out at the NSLS, Brookhaven National Laboratory, is supported by the Department of Energy, Material Sciences and Division of Chemical Sciences under Contract No. DE-AC02-76CH00016. One of us (I.M.T.) would like to acknowledge partial financial support from NATO.
*Present address: Department of Physics, Brookhaven National Laboratory, Upton, NY 11973.

${ }^{1}$ A. H. Compton, Bull, Natl. Res. Counc. (U.S.) 20, 48 (1922).

${ }^{2}$ L. G. Parratt, Phys. Rev. 95, 359 (1954).

${ }^{3}$ B. C. Lu and S. A. Rice, J. Chem. Phys. 68, 5558 (1978).

${ }^{4}$ D. Sluis and S. A. Rice, J. Chem. Phys. 79, 5658 (1983).

${ }^{5}$ L. Bosio and M. Oumezine, J. Chem. Phys. 80, 959 (1984).

${ }^{6}$ L. Bosio, R. Cortes, A. Defrain, and M. Oumezine, J. NonCryst. Solids 61\&62, 697 (1984).

${ }^{7}$ R. A. Cowley and T. W. Ryan, J. Phys. D 20, 61 (1987).

${ }^{8}$ M. Pomerantz, A. Segmuller, L. Netzer, and J. Sagiv, Thin Solid Films 132, 153 (1985).

${ }^{9}$ M. Pomerantz and A. Segmuller, Thin Solid Films 68, 33
(1980).

${ }^{10}$ R. M. Richardson and S. J. Roser, Liq. Cryst. 2, 797 (1987).

${ }^{11} \mathrm{~J}$. Als-Nielsen, F. Christensen, and P. S. Pershan, Phys. Rev. Lett. 48, 1107 (1982).

${ }^{12}$ P. S. Pershan and J. Als-Nielsen, Phys. Rev. Lett. 52, 759 (1984).

${ }^{13}$ B. M. Ocko, A. Braslau, P. S. Pershan, J. Als-Nielsen, and M. Deutsch, Phys. Rev. Lett. 57, 94 (1986).

${ }^{14}$ P. S. Pershan, A. Braslau, A. H. Weiss, and J. Als-Nielsen, Phys. Rev. A 35, 4800 (1987).

${ }^{15}$ P. S. Pershan, Proc. Natl. Acad. Sci. 84, 4692 (1987).

${ }^{16}$ E. F. Gramsbergen, W. H. de Jeu, and J. Als-Nielsen, J. Phys. (Paris) 47, 711 (1986). 
${ }^{17}$ D. K. Schwartz, A. Braslau, B. M. Ocko, and P. S. Pershan, Phys. Rev. A 38, 8457 (1988).

${ }^{18}$ A. Braslau, M. Deutsch, P. S. Pershan, A. H. Weiss, J. AlsNielsen, and J. Bohr, Phys. Rev. Lett. 54, 114 (1985).

${ }^{19}$ A. B. Braslau, P. S. Pershan, G. Swislow, B. M. Ocko, and J. Als-Nielsen, Phys. Rev. A 38, 2457 (1988).

${ }^{20}$ F. Rieutord, J. J. Benattar, and L. Bosio, J. Phys. (Paris) 47, 1249 (1986)

${ }^{21}$ S. G. Wolf, L. Leiserowitz, M. Lahav, M. Deutsch, K. Kjaer, and J. Als-Nielsen, Nature 328, 63 (1987).

${ }^{22}$ C. A. Helm, H. Möhwald, K. Kjaer, and J. Als-Nielsen, Europhys. Lett. 4, 697 (1987).

${ }^{23}$ B. M. Ocko and S. J. G. Mochrie, Phys. Rev. B 38, 7378 (1988).

${ }^{24}$ D. Gibbs, B. M. Ocko, D. M. Zehner, and S. J. G. Mochrie, Phys. Rev. B 38, 7303 (1988).

${ }^{25}$ K. B. Blodgett and I. Langmuir, Phys. Rev. 51, 964 (1937).

${ }^{26} \mathrm{~J}$. D. Swalen et al., Langmuir 3, 932 (1987).

${ }^{27}$ J. Sagiv, J. Am. Chem. Soc. 102, 92 (1980).

${ }^{28}$ L. Netzer, R. Iscovici, and J. Sagiv, Thin Solid Films 99, 235 (1983).

${ }^{29}$ L. Netzer, R. Iscovici, and J. Sagiv, Thin Solid Films 100, 67 (1983).

${ }^{30}$ R. Maoz and J. Sagiv, J. Colloid Interface Sci. 100, 465 (1984).

${ }^{31}$ E. Sabatani, R. Maoz, J. Sagiv, and I. Rubinstein, J. Electro. Anal. Chem. Interfacial Electrochem. 219, 365 (1987).

${ }^{32}$ S. R. Cohen, R. Naaman, and J. Sagiv, Phys. Rev. Lett. 58, 1208 (1987).

${ }^{33}$ W. A. Zisman, Adv. Chem. Ser. 43, 1 (1964).

${ }^{34}$ S. R. Wasserman, G. M. Whitesides, I. M. Tidswell, B. M. Ocko, P. S. Pershan, and J. D. Axe, J. Am. Chem. Soc. 111, 5852 (1989).

${ }^{35}$ D. W. Oxtoby and S. A. Rice, J. Chem. Phys. 76, 5278 (1982).
${ }^{36}$ M. Born and E. Wolf, Principles of Optics, 6th ed. (Pergamon, New York, 1980).

${ }^{37}$ J. Als-Nielsen, Physica 140A, 376 (1986).

${ }^{38}$ Y. Yoneda, Phys. Rev. 131, 2010 (1963).

${ }^{39}$ S. K. Sinha, E. B. Sirota, S. Garoff, and H. B. Stanley, Phys. Rev. B 38, 2297 (1988).

${ }^{40}$ S. R. Wasserman, Y-T. Tao, and G. M. Whitesides, Langmuir 5, 1074 (1989)

${ }^{41}$ C. Tanford, The Hydrophobic Effect: Formation of Micelles and Biological Membranes (Wiley, New York, 1973), p. 52.

${ }^{42}$ The Physics of $\mathrm{SiO}_{2}$ and its Interfaces, edited by K. Sokrates and T. Pantelides (Pergamon, New York, 1978).

${ }^{43}$ B. E. Warren, $X-R a y$ Diffraction (Addison-Wesley, Reading, MA, 1969), p. 108.

${ }^{44}$ S. C. Nyburg and H. Lüth, Acta Crystallogr. B 28, 2992 (1972).

${ }^{45}$ G. L. Gaines, Jr., Insoluble Monolayers at Liquid-Gas Interfaces (Interscience, New York, 1966), and references cited therein.

${ }^{46}$ S. R. Cohen, R. Naaman, and J. Sagiv, J. Phys. Chem. 90, 3054 (1986).

${ }^{47}$ S. Garoff, R. B. Hall, H. W. Deckman, and M. S. Alvarez, Proc. Electron. Chem. Soc. 112, 85 (1985).

${ }^{48}$ S. R. Andrews and R. A. Cowley, J. Phys. C 55, 6427 (1986).

${ }^{49}$ I. K. Robinson, Phys. Rev. B 33, 3830 (1986).

${ }^{50}$ A. Ourmazd, D. W. Taylor, J. A. Rentschler, and J. Bevk, Phys. Rev. Lett. 59, 213 (1987).

${ }^{51} \mathrm{~A}$. Ourmazd and J. Bevk, The Structure of the $\mathrm{Si} / \mathrm{SiO}_{2}$ Interface: A Review, Vol. 105 of Materials Research Society Symposium Proceedings: $\mathrm{SiO}_{2}$ and Its Interfaces, edited by S. T. Pantelides and G. Luovsky (Materials Research Society, Pittsburgh, 1988), p. 1. 